\title{
Formes temporelles de l'incertitude et sécurisation des trajectoires dans l'insertion professionnelle des jeunes
}

Uncertainty and job trajectory in young people access to the labour market

Henri Eckert et Virginie Mora

\section{OpenEdition}

Journals

Édition électronique

URL : http://journals.openedition.org/travailemploi/4341

DOI : 10.4000/travailemploi.4341

ISSN : 1775-416X

Éditeur

DARES - Ministère du Travail

Édition imprimée

Date de publication : 17 mars 2008

Pagination : $31-57$

ISSN : 0224-4365

\section{Référence électronique}

Henri Eckert et Virginie Mora, «Formes temporelles de l'incertitude et sécurisation des trajectoires dans l'insertion professionnelle des jeunes », Travail et Emploi [En ligne], 113 | janvier-avril 2008, mis en ligne le 17 février 2011, consulté le 19 avril 2019. URL : http://journals.openedition.org/ travailemploi/4341 ; DOI : 10.4000/travailemploi.4341 


\title{
Formes temporelles de l'incertitude et sécurisation des trajectoires dans l'insertion professionnelle des jeunes
}

\author{
Henri Eckert, Virginie Mora (*)
}

Les auteurs exploitent l'enquête "Génération 1998 en 2005 », particulièrement les calendriers détaillés des emplois successivement occupés au cours des premières années de la vie active par cette génération. Ils étudient la précarité à laquelle beaucoup ont été confrontés à la fin du cycle des études: serait-elle l'effet d'un ajustement de l'offre et de la demande d'emploi qui se ferait à leur détriment, en les cantonnant longtemps à la périphérie de l'emploi continu? L'étude montre la diversité des trajectoires et des formes de précarité, parfois paradoxales, que connaissent les jeunes, indépendamment du sexe, du niveau de diplôme ou de la spécialité. L'incertitude sur le marché du travail ne se réduit plus à une opposition entre contrats permanents et contrats temporaires; l'enjeu d'une sécurisation des trajectoires serait de favoriser une continuité susceptible de garantir contre les risques potentiels de dérive et de décrochage de l'emploi.

Le mot «précarité» s'est progressivement imposé pour désigner, dans le discours ordinaire, l'incertitude qui affecte la situation tant économique que sociale de certaines catégories de salariés. Cette incertitude survient, dans le contexte actuel d'effritement du compromis salarial (CASTEL, 1995), lorsque l'individu ou son groupe d'affiliation ne sont plus en mesure de préserver des conditions d'emploi jugées satisfaisantes. En d'autres termes: lorsqu'il leur devient difficile de se maintenir dans des emplois susceptibles, tout à la fois, de leur fournir les gratifications matérielles et symboliques qui peuvent en être légitimement attendues et de leur garantir une maîtrise suffisante sur l'avenir. Ou encore: lorsqu'il devient difficile d'obtenir ou de conserver, pour rester dans le langage courant, un «bon emploi» et/ou une «bonne situation» ou, pour reprendre cette fois les termes de Serge PaUgam, lorsque «l'intégration professionnelle» n'est plus «assurée» (2000).

Cette incertitude semble peser davantage sur les trajectoires professionnelles et sociales de certaines catégories : avant tout ceux qui, quel que soit leur âge ou leur sexe, ne disposent pas d'une formation professionnelle reconnue, aisément valorisable sur le marché du travail; mais aussi les femmes, plus souvent contraintes à l'emploi à temps partiel non choisi que leurs collègues hommes; ou les

$(*)$ Cereq, Centre d'études et de recherches sur les qualifications; mora@cereq.fr; eckert@cereq.fr. travailleurs plus âgés, en particulier lorsque, la cinquantaine entamée, le chômage les confronte aux vicissitudes du marché du travail et aux difficultés pour retrouver un nouvel emploi; les jeunes enfin, à l'autre extrémité du cycle de vie professionnelle, dont l'entrée dans l'activité se fait de plus en plus souvent sous le signe de l'emploi à durée déterminée, notamment lorsqu'ils sont contraints à courir les agences d'intérim pour obtenir une embauche. Si la "précarité juvénile» peut alors susciter des «stratégies d'adaptation» (NICOLE-DrancourT, 1992) à la situation sur le marché de l'emploi, elle n'en continue pas moins d'inquiéter les jeunes, leurs parents ou les pouvoir publics.

Car l'incertitude prend, dans le cas de la précarité juvénile, une tournure particulière: elle n'est généralement pas le résultat malencontreux d'un événement fâcheux, survenu en cours de vie professionnelle. Elle se condense plutôt dans la perspective de difficultés d'accès à l'emploi durable, difficultés d'autant plus prévisibles que les aînés les plus proches en ont fait déjà l'expérience. Ce n'est donc pas la perte de l'emploi ou la restriction des revenus qui inaugurent la période d'incertitude. C'est la fin d'une étape dans le cycle de vie - en l'occurrence la fin des études - qui introduit à une phase de turbulences d'autant plus probables que les individus concernés savent, ou pressentent, que leur installation dans un avenir assuré - un emploi pérenne et la sécurité qui en découle - leur demandera du temps. Cette durée dépend pour une grande part des ressources dont ils disposent et des aléas sur le marché du travail. En somme: l'incertitude 
procède ici davantage de sa «prévisibilité»(1), elle confine dans un «au jour le jour» (LECLERC-Olive, ENGRAND, 2000), sinon un «no future», et se traduit par diverses formes de «galère» (DUBET, 1987).

C'est à cette précarité-là, à laquelle nombre de jeunes-pas tous! - sont confrontés lorsqu'ils entrent sur le marché du travail, que nous nous intéresserons ici. Près de deux individus sur trois sortis de formation initiale en 2001, par exemple, ont débuté leur carrière professionnelle avec un contrat de travail temporaire alors que près d'un tiers de ceux qui étaient en emploi trois ans plus tard ne disposaient toujours que d'un tel contrat (CEREQ, 2005). Pour mieux saisir cette précarité généralement anticipée, nous avons opté pour l'observation longitudinale: repérer les successions d'états propres à l'insertion professionnelle constitue une condition pour en évaluer les dynamiques et cerner les formes temporelles de l'incertitude dans les parcours. Dans cette optique nous avons retenu deux critères spécifiques : non seulement le statut des emplois successivement occupés mais aussi la plus ou moins grande continuité de la relation d'emploi. Ces deux critères n'ont aucune prétention à définir la précarité(2), ils nous fournissent plus simplement un moyen d'analyser la temporalité de l'insertion professionnelle d'une cohorte donnée.

Après avoir explicité les deux critères retenus et la manière dont nous en ferons un usage combiné, nous tenterons de mettre en évidence, du point de vue de leur croisement, la diversité des trajectoires suivies par des individus sortis de formation initiale en 1998 ( $c f$. encadré 1$)$ et dont les parcours d'entrée dans la vie active ont été enregistrés sur une période de sept ans. C'est alors seulement que nous pourrons revenir sur les formes de l'expérience de la précarité faite par les jeunes qui entrent dans un marché du travail bouleversé par le développement des situations d'emploi particulières (FouRCADE, 1992; Maruani, Reynaud, 2004).

(1) Les jeunes sortis de formation initiale en 2001 ont pu - tout comme l'observateur extérieur informé, par exemple, des modalités de l'insertion professionnelle des jeunes sortis de formation en 1998 (CEREQ, 2002) - anticiper les conditions de leur insertion professionnelle. Ces anticipations prennent appui sur les circonstances dans lesquelles leurs aînés, grands frères, grandes sœurs ou leurs proches un peu plus âgés sont entrés dans la vie active, différentes de celles qu'a connues la génération précédente. Etayées sur des tendances de long terme, elles n'excluent pas l'imprévisible, les variations de la conjoncture en particulier.

(2) Le type du contrat de travail et la durée de l'emploi ne suffisent certes pas à définir la précarité d'une situation d'emploi. Bernard FourCADE (1992) considère que trois autres aspects au moins de la relation d'emploi doivent être pris en compte: sa discontinuité, le revenu et la protection sociale assurés par l'emploi. Mais lorsque la durabilité de l'emploi contredit le contrat temporaire, la précarité peut aussi être mise en doute (RAMAUX, 2006).

\section{Encadré 1}

\section{Génération 1998 en 2005}

L'enquête Génération 1998 en 2005 a permis de réinterroger un échantillon d'individus parmi ceux qui, sortis du système de formation initiale en 1998, avaient été interrogés une première fois en 2001 et une deuxième fois en 2003. Plus précisément:

«Pour constituer le panel Génération 1998, un échantillon de 33000 individus a été extrait des 54000 ayant répondu à la première interrogation au printemps 2001. Sur cet échantillon, 22000 jeunes ont répondu à la deuxième interrogation en 2003 et 16000 à la troisième interrogation en 2005 [...]. (1)

Les individus enquêtés lors des réinterrogations du panel présentent des caractéristiques différentes de ceux enquêtés lors de la première interrogation. En effet, il apparaît que les jeunes au chômage, vivant seuls au moment de la première interrogation ou issus de bas niveaux de qualifications ont été en proportion moins nombreux à répondre aux réinterrogations.

Pour mesurer les distorsions de la composition de l'échantillon entre les vagues successives d'interrogation, une analyse discriminante, de type régression logistique, a été réalisée afin de connaître la probabilité, pour les 33000 jeunes ayant répondu à la première interrogation et retenus dans le panel, de répondre lors de la troisième interrogation. Les distorsions significatives ont ainsi pu être redressées grâce à une pondération qui restitue la structure initiale du panel. » (CereQ, 2007)

(1) Plus précisément: lors de la deuxième interrogation, menée auprès de 33000 individus, 22000 réponses ont été obtenues dans les délais impartis; lors de la troisième interrogation, 16000 réponses ont été obtenues, toujours dans les délais impartis, auprès des 22000 répondants de la deuxième vague.

\section{Formes temporelles} de l'incertitude et régimes de participation au marché du travail

Après avoir défini les formes temporelles de l'incertitude à laquelle certains jeunes sont confrontés en début de vie active, nous montrerons comment celles-ci nous permettent de distinguer entre divers régimes de participation au marché du travail.

\section{Approcher les formes temporelles de l'incertitude}

L'accès à une situation d'emploi pérenne - dont la représentation courante se cristallise autour d'un emploi unique, sur contrat à durée indéterminée, obtenu dans une entreprise qui offre des garanties satisfaisantes de durabilité - suppose d'établir une relation d'emploi continue avec un ou éventuellement plusieurs employeurs, sans détour par le chômage dans ce dernier cas. Or la continuité dans le temps de la relation d'emploi s'évalue généralement à l'aune de deux critères, l'un relatif à la qualité du contrat de 
travail et l'autre à la durée effective en emploi. Ce second critère ne peut être mesuré, du fait de sa nature même, qu'après coup, lorsque l'emploi s'avère avoir duré un certain temps tandis que le premier constitue une sorte d'anticipation sur l'avenir. Que cette anticipation ne se concrétise pas toujours (3) n'enlève rien au fait que le contrat à durée indéterminée ait acquis une valeur sociale symbolique d'assurance a priori contre l'incertitude. La mesure de la durée effective de la relation d'emploi présente, quant à elle, l'avantage d'un constat vérifiable et fournit un critère a posteriori d'appréciation de la situation d'emploi, débarrassé de toute représentation sociale. N'y aurait-il pas alors un intérêt à croiser ces deux critères pour analyser après coup l'insertion d'une cohorte de jeunes dans la vie active? Prenons toutefois le temps de préciser un peu plus nos deux critères avant de revenir sur leur croisement.

Nous distinguerons ici deux types d'emplois selon la qualité juridique du contrat de travail: les emplois à durée déterminée (Edd) et les emplois à durée indéterminée (Edi). Ces derniers regroupent essentiellement des emplois conformes à la norme, instaurée durant les Trente glorieuses, d'emploi sans échéance fixée au moment de l'établissement du contrat. Les premiers rassemblent à l'inverse les formes d'emploi dérogatoires à cette norme, c'est-à-dire les emplois dont le terme est prévu dans le contrat de travail (Maruani, Reynaud, 2004). Ces formes d'emploi dérogatoires incluent les emplois sur contrat à durée déterminée, l'intérim, les différents types d'emplois aidés et stages, en somme l'ensemble des «formes particulières d'emploi» (id.) introduites depuis la fin des années 1970(4). Nous avons de même distingué deux catégories de situations vis-à-vis de l'emploi selon la continuité de la relation d'emploi: nous considérerons ici que la durabilité/continuité de la relation d'emploi est établie dès lors que l'individu n'a connu aucune interruption d'emploi pendant une durée minimale de dix-huit mois, quel que soit le nombre d'emplois qu'il a occupé pendant la période et quels que soient les contrats sur lesquels il était ou est toujours employé. Ce choix, s'il reste in fine arbitraire, s'appuie toutefois sur la durée maximale théorique d'un emploi sur contrat à durée déterminée

(3) L'embauche sur contrat à durée indéterminée n'assure pas toujours la pérennité de l'emploi. Certains secteurs économiques en font, par exemple, un usage qui vise davantage à contourner les obligations attachées aux contrats à durée déterminée (LOCHET, 1994).

(4) Conformément à ce point de vue, nous avons intégré, dans les analyses qui suivent, les quelques cas d'installation à son compte ou les emplois d'aide familiaux au premier groupe, même si ces derniers, par exemple, constituaient le type même de l'emploi précaire dans les années 1960. De toute façon leur petit nombre n'a pas d'incidence significative sur nos résultats. ou d'une mission d'intérim(5). On peut risquer l'hypothèse selon laquelle une grande part des individus qui réussissent à se maintenir en emploi continûment pendant une période de plus de dix-huit mois tendrait ainsi vers une certaine assurance quant à leur avenir.

Le croisement de ces deux critères permet alors de constituer quatre classes d'états vis-à-vis de l'emploi: dans deux cas de figure les deux critères paraissent diverger alors qu'ils semblent converger dans les deux autres. Il en va ainsi lorsque les individus occupent, au mois considéré, un Edd et n'ont pas réussi à accumuler dix-huit d'emploi continu (état $\mathrm{A}, c f$. tableau 1) ou lorsqu'ils ont, à l'inverse, obtenu d'être employés sur un Edi et qu'ils sont continûment en emploi depuis dix-huit mois au moins (état D). En revanche, lorsque des individus qui occupent un Edd ont pourtant accumulé au moins dix-huit mois d'emploi sans interruption (état B) ou lorsqu'ils n'ont pas réussi à se maintenir aussi longuement en emploi tout en occupant un Edi (état C), les deux critères semblent effectivement diverger. Dans ce dernier cas de figure, il s'agit le plus souvent d'individus qui ont réussi à obtenir un emploi sur contrat à durée indéterminée mais n'ont, au mois considéré, pas encore passé dix-huit mois dans cet emploi. Pour la plupart, ils se maintiendront dans l'emploi et passeront à l'état $\mathrm{D}(6)$. La situation véritablement paradoxale est celle où l'individu est dans l'état B: employé sur un Edd, il parvient pourtant à se maintenir en emploi continûment(7).

Nous reviendrons plus loin sur cet état B, qui ne laisse pas d'intriguer, ou plutôt sur les trajectoires qui lui correspondent, d'autant plus que le nombre d'individus concernés ne saurait trouver sa raison dans les seuls contrats précaires qui excèdent la durée que nous avons arbitrairement retenue pour valider la continuité de la relation d'emploi. Leur proportion, si elle est divisée par deux entre 2000 et 2005, n'en reste pas moins conséquente. La part d'individus dans l'état $\mathrm{D}$ - emploi à durée indéterminée et continuité d'emploi supérieure à dix-huit mois - a, quant à elle, doublé dans le même intervalle de temps: cette évolution témoigne d'une pérennisation progressive de la situation profes-

(5) La durée maximale d'un emploi sur contrat à durée déterminée peut, dans certaines circonstances, être portée à vingtquatre mois et les emplois-jeunes étaient prévus pour une durée maximale de cinq ans... Et si le premier emploi occupé par les sortants de la Génération 1998 est deux fois sur trois un emploi temporaire, les emplois aidés représentent $12 \%$ des premiers emplois, dont un quart seulement, soit $3 \%$ des premiers emplois environ, sont des emplois-jeunes, susceptibles de durer jusqu'à cinq ans. Nous avons donc opté pour un seuil à dix-huit mois, dans la mesure où il constitue la limite d'une forte majorité d'emplois à durée déterminée.

(6) Cette propension se vérifie empiriquement par la fréquence des successions entre état $\mathrm{C}$ et état $\mathrm{D}$ au fil des trajectoires effectives des individus.

(7) Ces situations ne sont par rares sur le marché du travail; elles sont évoquées notamment dans les analyses et prises de position de C. RAMAUX (2006) sur la précarité. 
Tableau 1

Classes d'états vis-à-vis de l'emploi

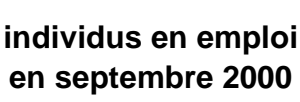

emploi à durée

déterminée

emploi à durée

indéterminée
Non-continuité

ancienneté d'emploi

inférieure à 18 mois
Continuité

ancienneté d'emploi

supérieure à 18 mois

\begin{tabular}{|cc|cc|}
\hline état A & état B & \\
& $16 \%(2576 \mathrm{Rnp})$ & & $16 \%(2590 \mathrm{Rnp})$ \\
\hline état C & & état D & \\
& $15 \%(2496 \mathrm{Rnp})$ & & $34 \%(5702 \mathrm{Rnp})$ \\
\hline
\end{tabular}

total des individus en emploi

81\% (13364 Rnp)

état Z : total des individus sans emploi $\quad 19 \%$ (2676 Rnp)

total $\mathbf{1 0 0 \%}(16040 \mathrm{Rnp})$

\section{individus en emploi en septembre 2005}

\section{emploi à durée déterminée}

emploi à durée indéterminée

\begin{tabular}{|c|c|}
\hline $\begin{array}{l}\text { Non-continuité } \\
\text { ancienneté d'emploi } \\
\text { inférieure à } 18 \text { mois }\end{array}$ & $\begin{array}{c}\text { Continuité } \\
\text { ancienneté d'emploi } \\
\text { supérieure à } 18 \text { mois }\end{array}$ \\
\hline $5 \%(788 \mathrm{Rnp})$ & 8\% (1176 Rnp) \\
\hline 5\% (750 Rnp) & 69\% (11472 Rnp) \\
\hline
\end{tabular}

total des individus en emploi

état Z : total des individus sans emploi

total
87\% (14186 Rnp)

13\% (1854 Rnp)

100\% (16040 Rnp)

effectif pris en compte : 740000 individus sortis de formation en 1998

effectif non-pondéré : 16040 individus

Les pourcentages en gras sont calculés sur les effectifs pondérés - Rnp : répondants non pondérés

sionnelle d'une part de plus en plus importante des jeunes sortis de formation en 1998, sans qu'il en aille pourtant de même pour la totalité de la cohorte. Alors que certains restent éloignés de l'emploi (état Z), un nombre important de jeunes de la cohorte subit toujours les aléas d'une intégration professionnelle que nous qualifierons - en reprenant certains termes de Serge PaUgam (2000), quitte à leur donner un sens plus ordinaire (8) -, sinon d'incertaine, du moins de laborieuse. Or la question se pose bel et bien de savoir si les formes de la participation de ces jeunes ne risquent pas, de même, de rester laborieuses encore un temps et, dans les pires des cas, incertaines ou disqualifiantes.

Ce sont donc les régimes de la participation au marché du travail des jeunes ici pris en exemple - ceux de la Génération 1998 ( $c f$. encadré 1) - qui nous intéressent. Avant de les évoquer, à travers l'analyse de leurs trajectoires, il importe toutefois de souligner une caractéristique inhérente à notre

(8) En effet, nous ne prenons pas en compte le critère de satisfaction au travail, combiné par Serge Paugam avec celui de stabilité/précarité pour définir sa typologie de l'intégration professionnelle; nous utilisons donc ici ses mots dans leur sens ordinaire, descriptif. approche qui nous place plutôt dans la perspective d'observer les modes de participation au marché du travail en début de vie active que le processus d'insertion proprement dit. Dans la mesure où nous voulions prendre en compte le critère de continuité de la relation d'emploi et comme nous avons fixé à dix-huit mois le terme au-delà duquel nous considérons que cette continuité est avérée, nous avons choisi de focaliser l'analyse sur la période 20002005. Du fait de la définition du seuil minimal de continuité de l'emploi, aucun individu ne peut, en effet, se prévaloir d'une ancienneté dans l'emploi suffisante pour atteindre ce seuil tant que dix-huit mois au moins ne se sont pas écoulés depuis la sortie de formation initiale (cf. graphique 1: il faut attendre le milieu sinon la fin de l'année 1999 pour que des individus se retrouvent dans les états B ou D). Notre analyse ne portera donc pas tant sur l'insertion immédiate de ces jeunes mais plutôt sur la période qui s'étend de la troisième à la septième année de leur vie active.

\section{Repérer les régimes de participation des jeunes au marché du travail}

Au fil de leur évolution dans la vie active, les jeunes changent d'état vis-à-vis de l'emploi, 
Graphique 1

\section{Situation vis à vis de l'emploi des sortants de la Génération 1998}

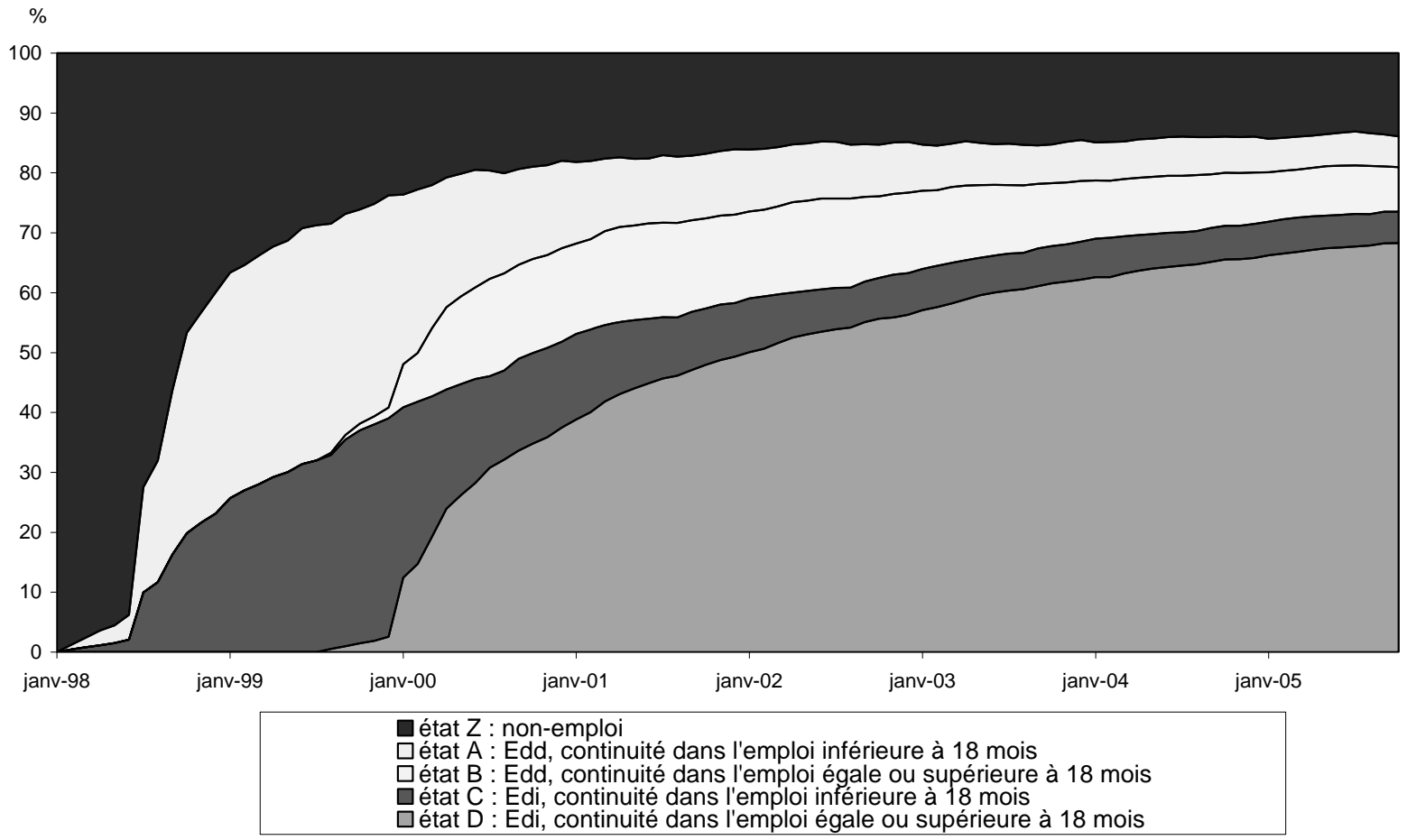

passant par exemple de l'état $\mathrm{A}$ à l'état $\mathrm{C}$ au bout de quelques mois pour se retrouver, quelques mois plus tard encore, dans l'état D. Si une telle trajectoire s'avère particulièrement rassurante, puisque l'individu débouche sur une situation d'emploi pérennisée, il n'en va pas toujours de même. L'examen des parcours repose ici sur une analyse en correspondances multiples (ACM), qui porte sur l'ensemble des trajectoires individuelles codées de mois en mois, entre septembre 2000 et septembre 2005, selon l'état vis-à-vis de l'emploi; une classification hiérarchique est ensuite effectuée à partir des vecteurs de coordonnées obtenus(9) et la mise en évidence de dix trajectoires typiques apparaît bien adaptée dans la mesure où cette partition capte près de la moitié de l'inertie du nuage initial. La césure à gauche obère certes l'influence, sur la classification obtenue, des premiers pas dans la vie active, lorsque les tâtonnements sont nombreux; mais notre classification ne vise pas une description de la totalité des trajectoires depuis la sortie de formation. Elle focalise sur la portion de trajectoire au cours de laquelle le critère de durabilité de la relation d'emploi peut être mis en œuvre ( $c f$. paragraphe précédent). À noter que la Génération 1998 est entrée sur le marché du travail dans un contexte conjoncturel relativement favorable, avantage que la dégradation économique ultérieure ne fait pas totalement disparaître ensuite.

Les changements d'emploi ne sont pas saisis lorsque deux emplois se succèdent sans interruption

(9) Trente-six vecteurs de coordonnées ont été retenus, de sorte que ceux-ci restituent environ $90 \%$ de l'inertie du nuage initial. de la participation à l'activité salariée et demeurent donc invisibles. En outre, certains aspects déterminants de la précarité restent inaperçus: qu'il s'agisse du salaire ou des avantages sociaux liés ou non à l'emploi, qu'il s'agisse des rapports entre la formation acquise par l'individu et les activités qui lui sont confiées dans le cadre de l'emploi, qu'il s'agisse de son niveau de formation, des espérances qui lui étaient liées et du niveau effectif de l'emploi occupé, qu'il s'agisse enfin du temps de travail et notamment de temps partiel imposé plutôt que souhaité, ces différents aspects de la relation salariale sont ici ignorés. Bien qu'ils puissent contribuer à la précarité des situations d'emploi, ils ne sont pas pris en compte dans la mesure où nous avons conçu un dispositif d'analyse de la précarité juvénile qui focalise sur la temporalité du processus d'insertion et s'attache davantage aux formes d'incertitude liées à la durabilité de la situation d'emploi. Nous vérifierons que ce choix permet de mettre en évidence des formes de continuité dans l'emploi là où les types de contrats de travail ne permettaient guère de prévoir sa durabilité ou encore des participations soutenues à l'activité malgré les discontinuités de l'emploi.

Nous nous intéressons donc ici aux régimes de participation des jeunes au marché du travail en début vie active. Car si une moitié de la Génération 1998 n'a guère connu de difficulté pour pérenniser sa situation professionnelle ( $c f$. ci-dessous trajectoires de type 1), les parcours sont moins linéaires pour l'autre moitié. Parmi cette seconde moitié, un jeune sur trois environ suit une trajectoire marquée par une discontinuité de sa présence en emploi, due généralement à une période de chômage plus ou moins longue, mais parvient finalement à s'ins- 


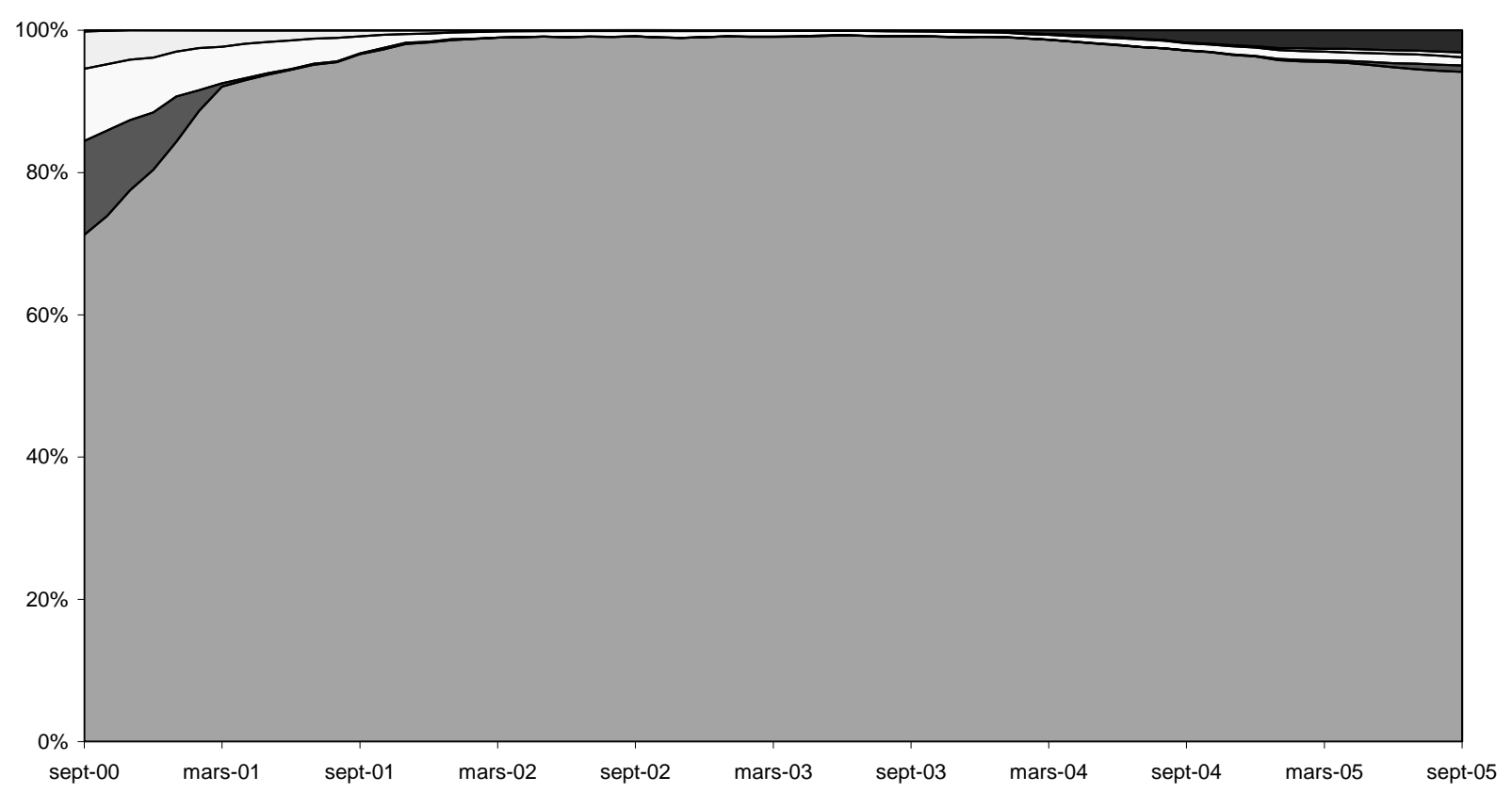

Graphique 3

Régime 1, trajectoire 2 : $9 \%$ des parcours

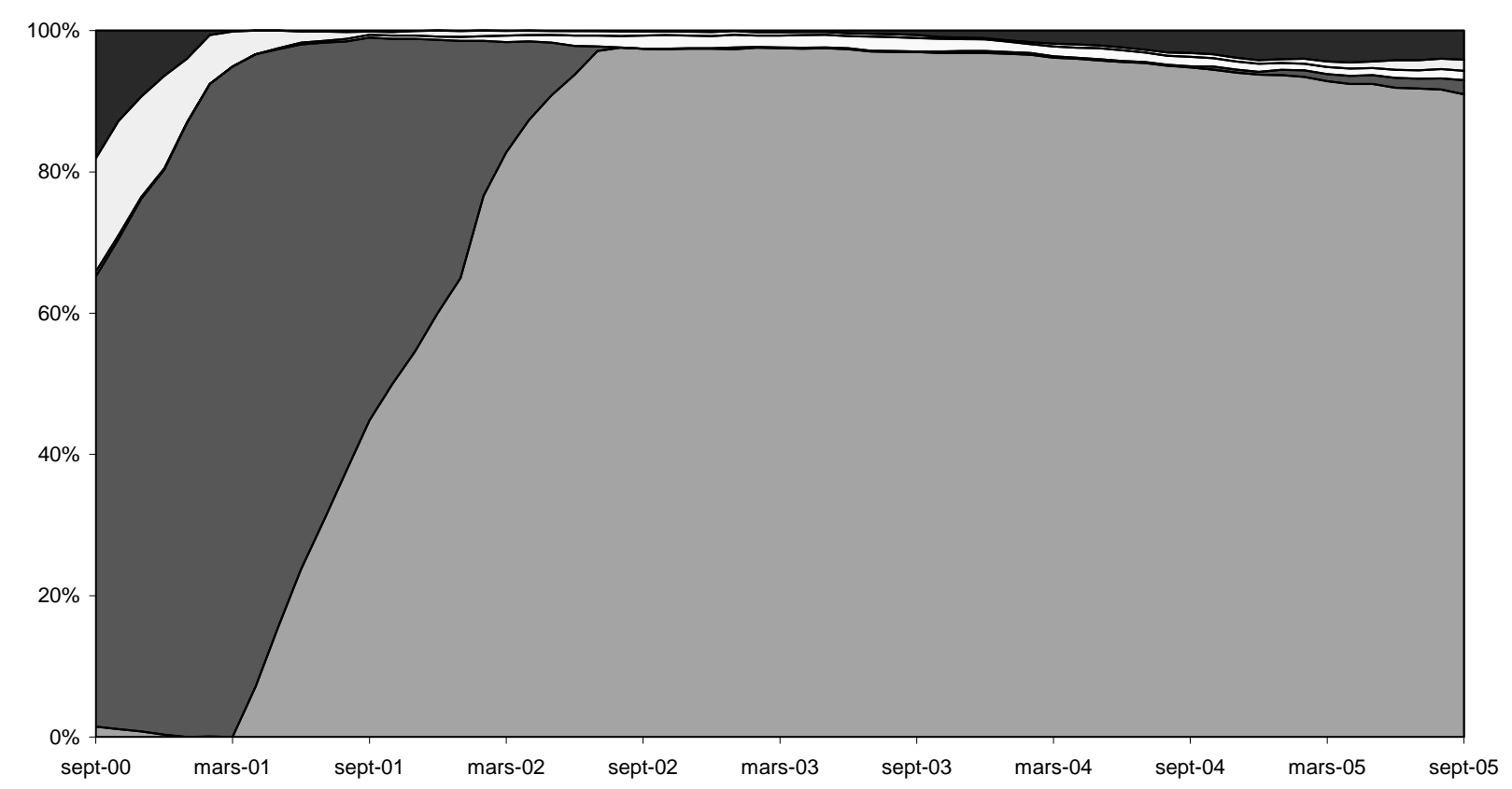

Voir légendes graphique 1.

taller durablement en emploi ( $c f$. trajectoires de type 2). Un autre parvient à se maintenir en emploi de manière plutôt continue sans pour autant accéder à un emploi sur contrat à durée indéterminée au cours de la période d'observation ou finit par accéder à un emploi sur contrat à durée indéterminée à l'issue d'une longue période d'emploi sous statut temporaire, sans détour par le non-emploi ( $c f$. trajectoires de type 3 ). Le dernier des trois, enfin, suit un parcours fortement discontinu, fait d'alternances emploi/non-emploi et réussit ou non, selon les cas, à préserver une participation importante à l'emploi ( $c f$. trajectoires de type 4). Détaillons ces différents cas de figure!

\section{Régime 1 : installation dans l'Edi sans anicroche notable}

Les trajectoires relevant de ce régime - suivies, rappelons-le, dans environ la moitié des parcours se caractérisent par une installation précoce dans l'emploi à durée indéterminée. L'inscription dans la 
continuité d'emploi, observée entre 2000 et 2005, a en effet commencé avant 2000 - elle est quasiment acquise dès 2000 pour ceux qui ont suivi la première trajectoire (graphique 2), encore en cours en début de période d'observation pour ceux qui ont suivi la seconde (graphique 3) - et elle perdure, que l'individu ait connu un ou plusieurs emplois successifs. Dans ce dernier cas, les emplois se suivent sans détour par le chômage. Ce régime renvoie ainsi à une participation au marché du travail qui se déroule sous le sceau de la continuité de l'emploi, dans le cadre de l'Edi, conforme à la représentation courante de la bonne insertion professionnelle et sociale.

\section{Régime 2 : installation dans l'Edi et pérennisation après quelques perturbations}

Trois trajectoires sont ici regroupées, qui représentent $17 \%$ des parcours. Ce régime de participation au marché du travail se caractérise par un passage hors de l'emploi plus ou moins long, à un moment de la trajectoire, mais cette période de nonemploi précède l'accès à un Edi durable ou susceptible de durer. Ces trajectoires se distinguent entre elles par le moment où survient l'installation dans l'emploi à durée indéterminée. Mais ce moment tient avant tout aux difficultés rencontrées en amont de cette installation dans l'emploi durable, selon que l'épisode de chômage - le «creux » qui infléchit ces trajectoires - survient en début de période d'observation, plutôt durant le premier trimestre 2001 (graphique 4), ou plus tardivement, aux abords du deuxième trimestre 2002 (graphiques 5), voire au cours du troisième trimestre 2003 (graphique 6). Il s'agit, en somme, de parcours marqués par des faux départs, des sorties de route accidentelles ou des périodes plus ou moins longues de flottement. $\mathrm{Ce}$ régime se caractérise ainsi par une perturbation $\mathrm{du}$ processus d'insertion professionnelle, momentanée mais a priori sans conséquence sur la durabilité ultérieure de la relation d'emploi.

\section{Régime 3: formes paradoxales de continuité d'emploi}

Ce régime renvoie à deux trajectoires qui, ensemble, représentent environ $16 \%$ des parcours. La première (graphique 7) rassemble des parcours de continuité dans l'emploi sur contrat à durée déterminée avant installation dans un emploi à durée indéterminée. En moyenne, entre 2000 et 2005 , la première moitié de la période s'écoule en emploi continu, auprès d'un seul employeur le plus souvent, mais sous statut temporaire. La seconde moitié de période consacre généralement l'accès à un statut permanent, mais les épisodes de chômage existent. Ces trajectoires correspondent parfois à celles de jeunes en formation par alternance, qui s'installent dans l'emploi durable par la suite, mais cette figure est loin d'épuiser le sujet. Près des trois quarts des jeunes de ce groupe n'ont connu aucune période de non-emploi après septembre 2000. La seconde trajectoire de ce régime (graphique 8) regroupe les itinéraires les plus paradoxaux: entre septembre 2000 et septembre 2005 , plus de $80 \%$ du temps s'est déroulé en Edd, sans interruption du lien à l'emploi. À l'automne 2005 la situation paradoxale perdure généralement. Le plus souvent, les années d'emploi continu sous statut temporaire ont été passées chez un seul et même employeur. S'il s'agit,

Graphique 4

Régime 2, trajectoire 1 : $7 \%$ des parcours

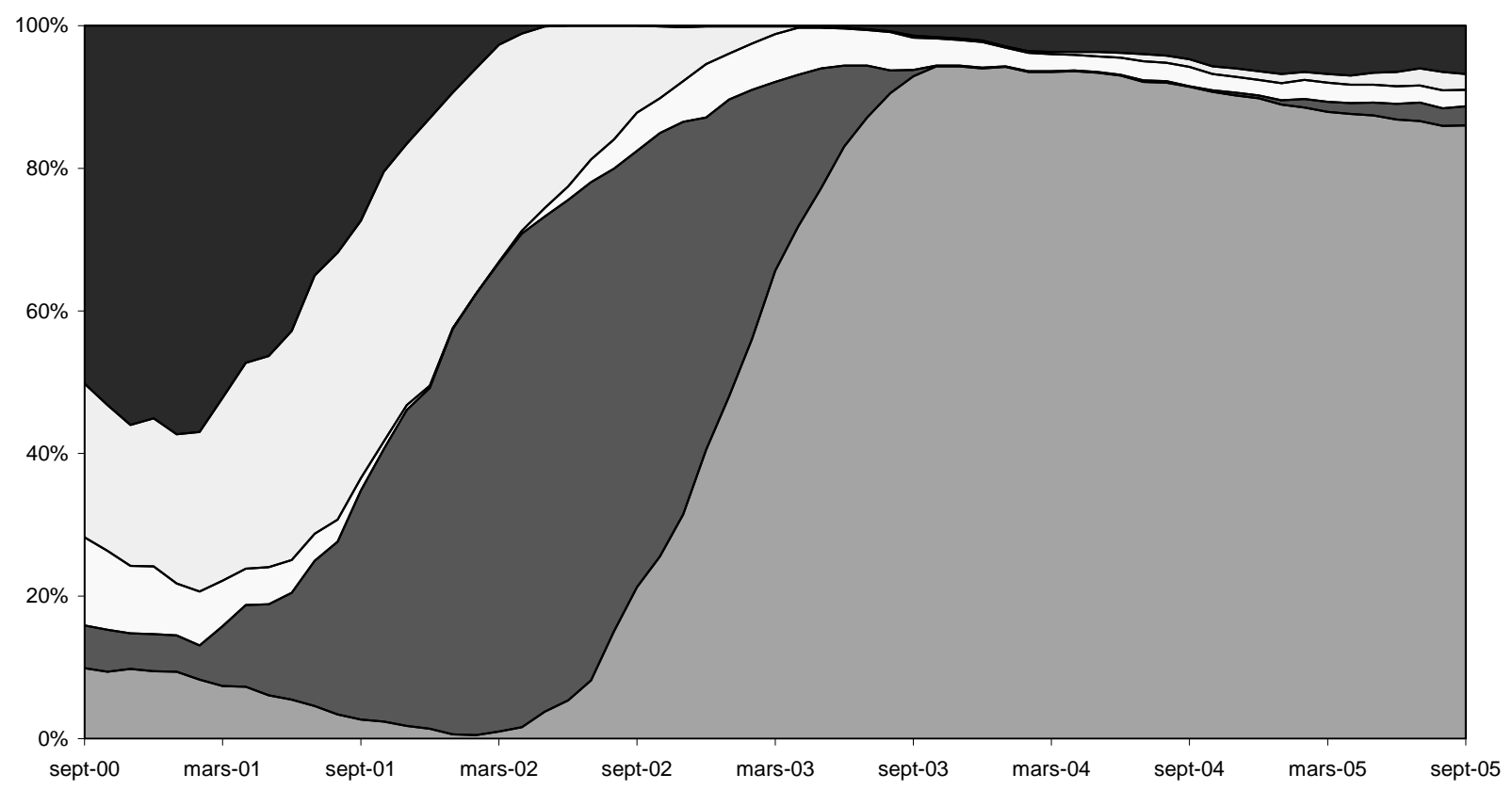


Régime 2, trajectoire 2 : $5 \%$ des parcours

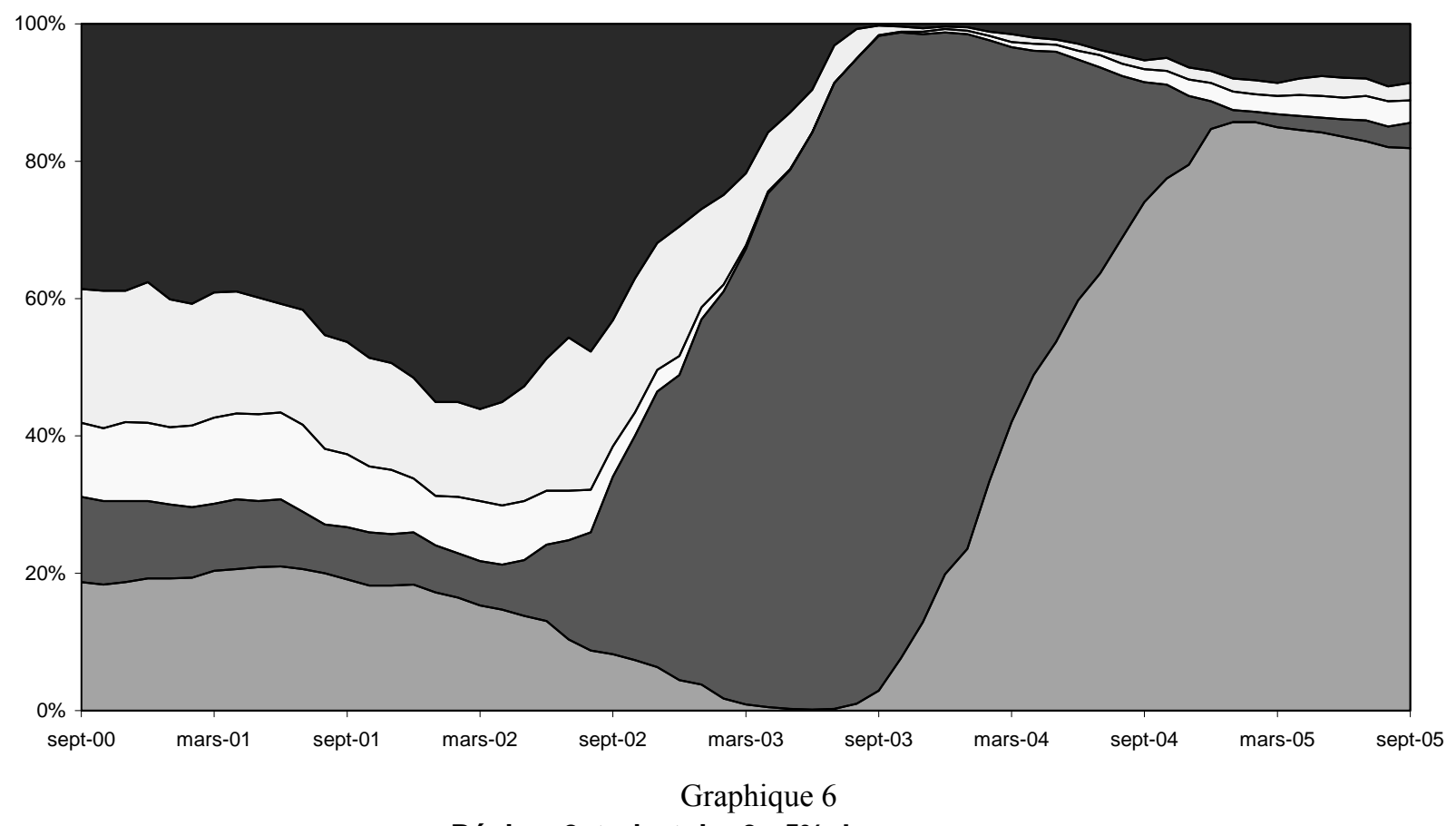

Régime 2, trajectoire 3 : $5 \%$ des parcours

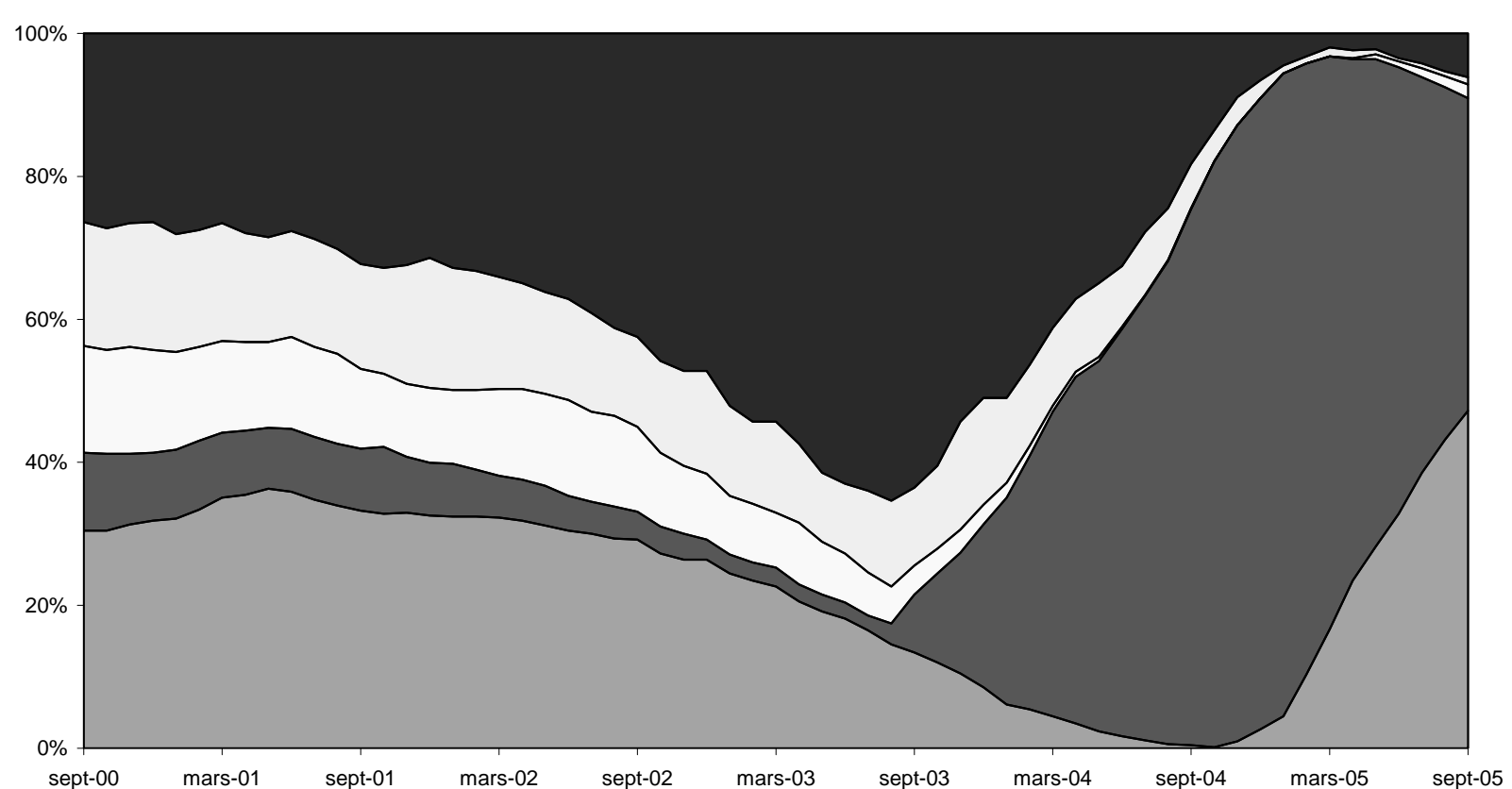

Voir légendes graphique 1.

dans quelques cas, d'emplois-jeunes, la plus grande part de ces situations échappe aux emplois aidés. C'est tout le paradoxe de ce régime de participation au marché du travail, qui lie la continuité d'emploi à des conditions d'emploi a priori précaires.

\section{Régime 4 : inscription dans l'emploi discontinu ou le non-emploi}

Les trajectoires caractéristiques de ce régime représentent environ $18 \%$ des trajectoires observées. Dans la première (graphique 9), la majorité du temps se déroule en emploi entre 2000 et 2005, plus souvent sous contrat temporaire, même si le contrat est parfois à durée indéterminée. Pour autant, $60 \%$ de ces parcours comportent au moins quatre emplois distincts, d'une durée médiane de douze mois, souvent séparés par des périodes de nonemploi. Dans la deuxième trajectoire (graphique 10), si le temps passé en emploi reste prépondérant entre 2000 et 2005, les périodes d'emploi sont entrecoupées de passages par le chômage. Si l'on compte également quatre emplois en moyenne, d'une durée médiane de neuf mois cette fois, un peu plus courte que précédemment, l'essentiel du temps se déroule en alternance entre emploi et non-emploi 
Graphique 7

Régime 3, trajectoire 1 : $9 \%$ des parcours

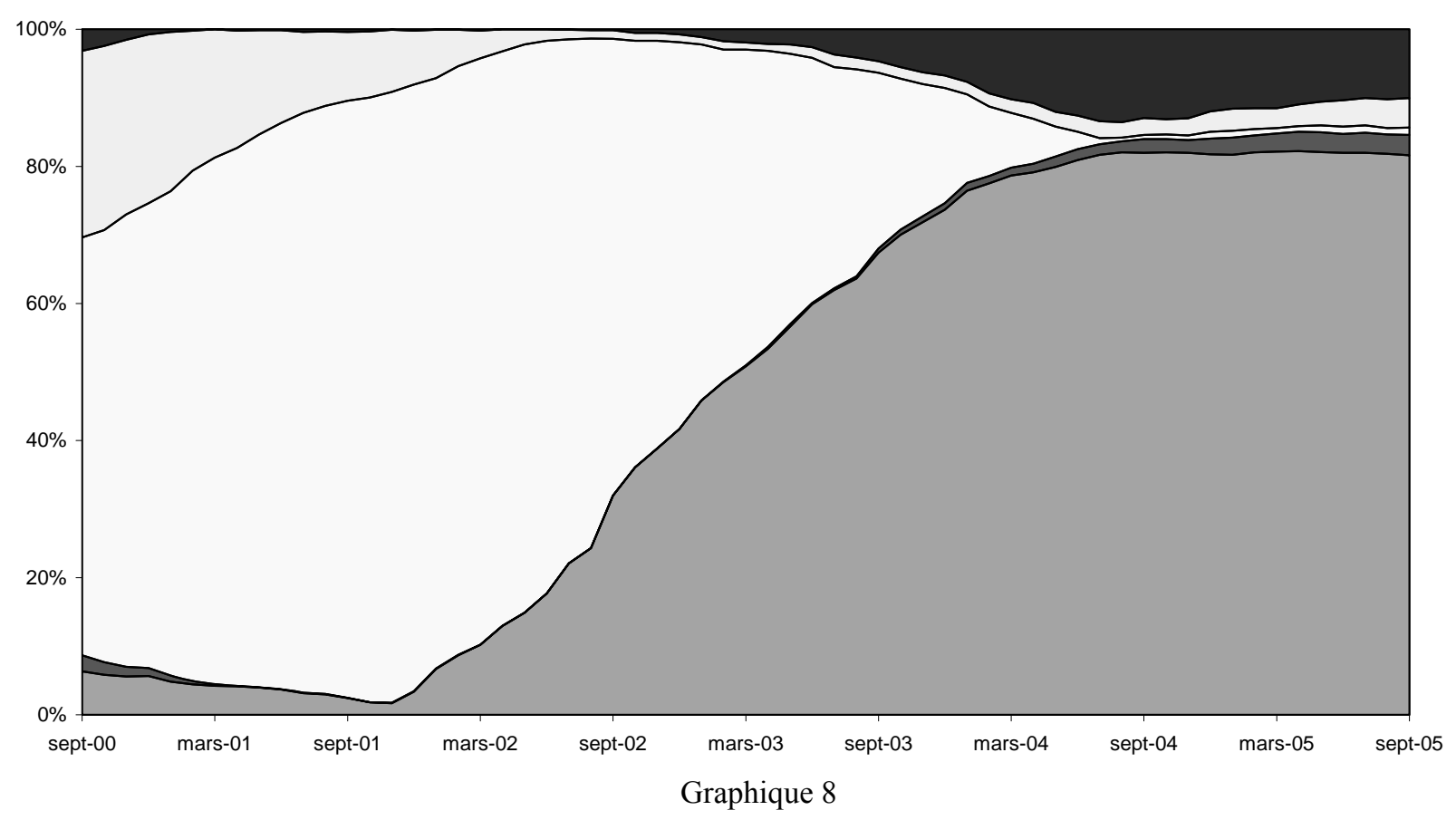

Régime 3, trajectoire 2: $7 \%$ des parcours

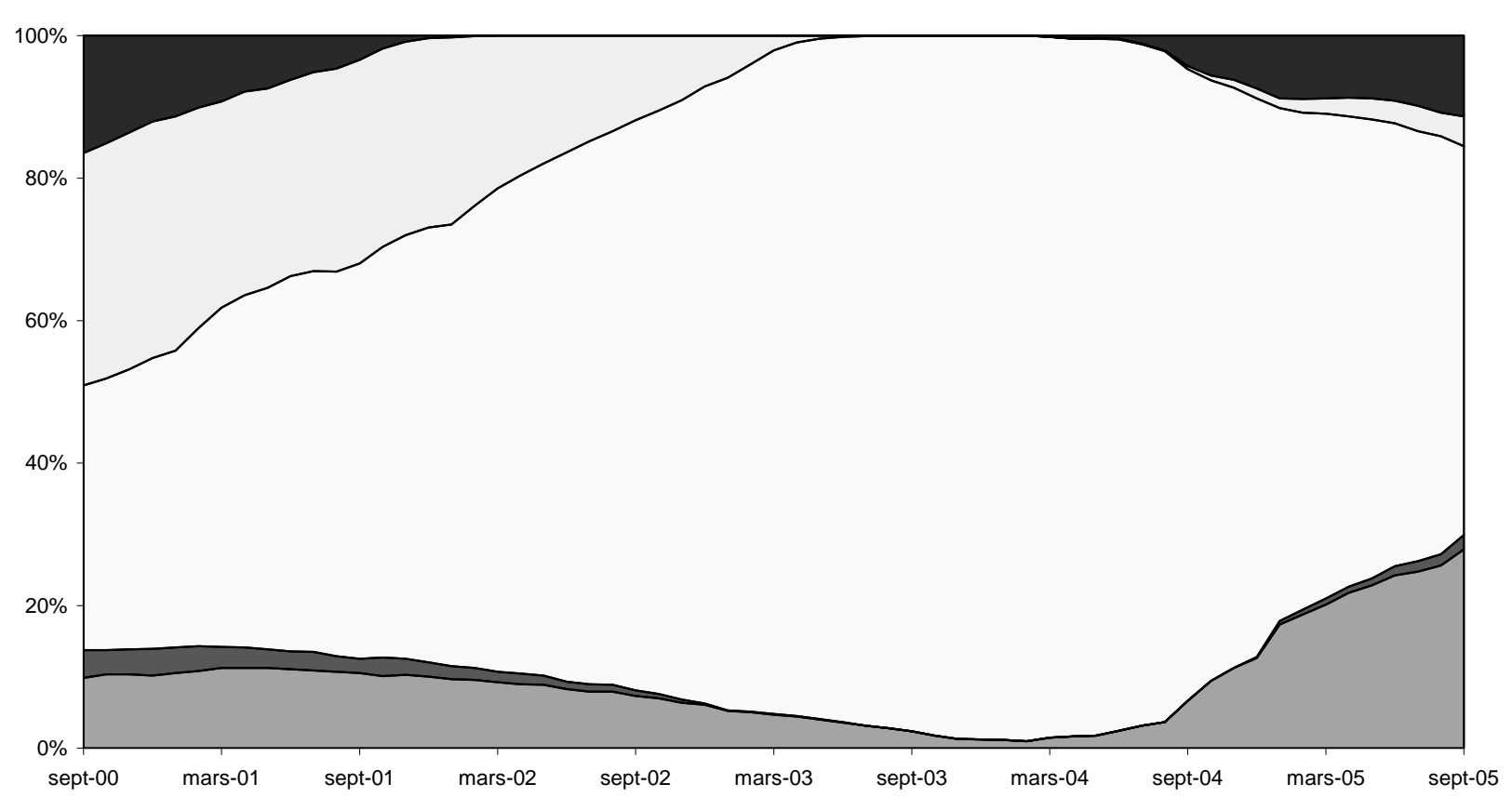

Voir légendes graphique 1.

et les accès à l'emploi durable en fin de période restent incertains. La troisième trajectoire enfin (graphique 11), regroupe des parcours marqués par une faible participation à l'emploi: près du quart des jeunes concernés n'ont même eu aucun emploi entre 2000 et 2005 . Un sur six est certes passé par un Edi entre 2000 et 2003 mais pour aboutir au chômage en fin de parcours. Les individus qui ont suivi cette trajectoire ont passé, en moyenne, quatre années hors de l'emploi. C'est ainsi la rareté ou la discontinuité de la relation d'emploi qui caractérise ce régime de participation au marché du travail.
Cette typologie des régimes de participation au marché du travail des nouveaux venus dans la vie active nous rappelle d'abord qu'une majorité des jeunes de la Génération 1998 n'ont, tous niveaux confondus, guère connu de difficultés pour s'installer dans l'emploi à durée indéterminée. Leur proportion augmente encore si, à ceux qui accèdent assez directement à l'emploi durable, sont ajoutés ceux qui retrouvent ce type d'emploi après un accident qui a interrompu momentanément leur trajectoire d'emploi. Au-delà de ce premier constat, la typologie suggère encore que le recours aux Edd longs, 
Graphiques 9, 10, 11

Régime 4, trajectoire 1 : $5 \%$ des parcours

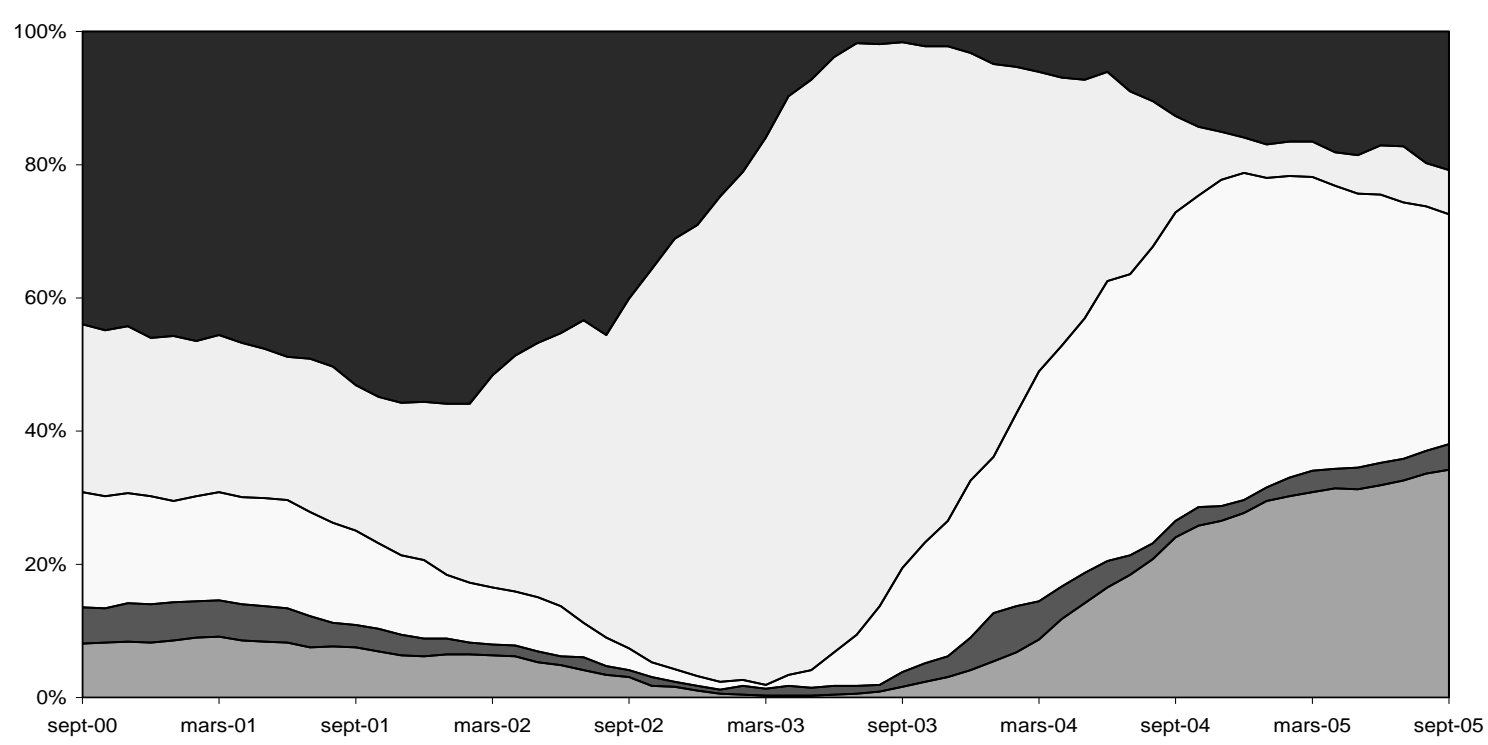

Régime 4, trajectoire 2 : $5 \%$ des parcours

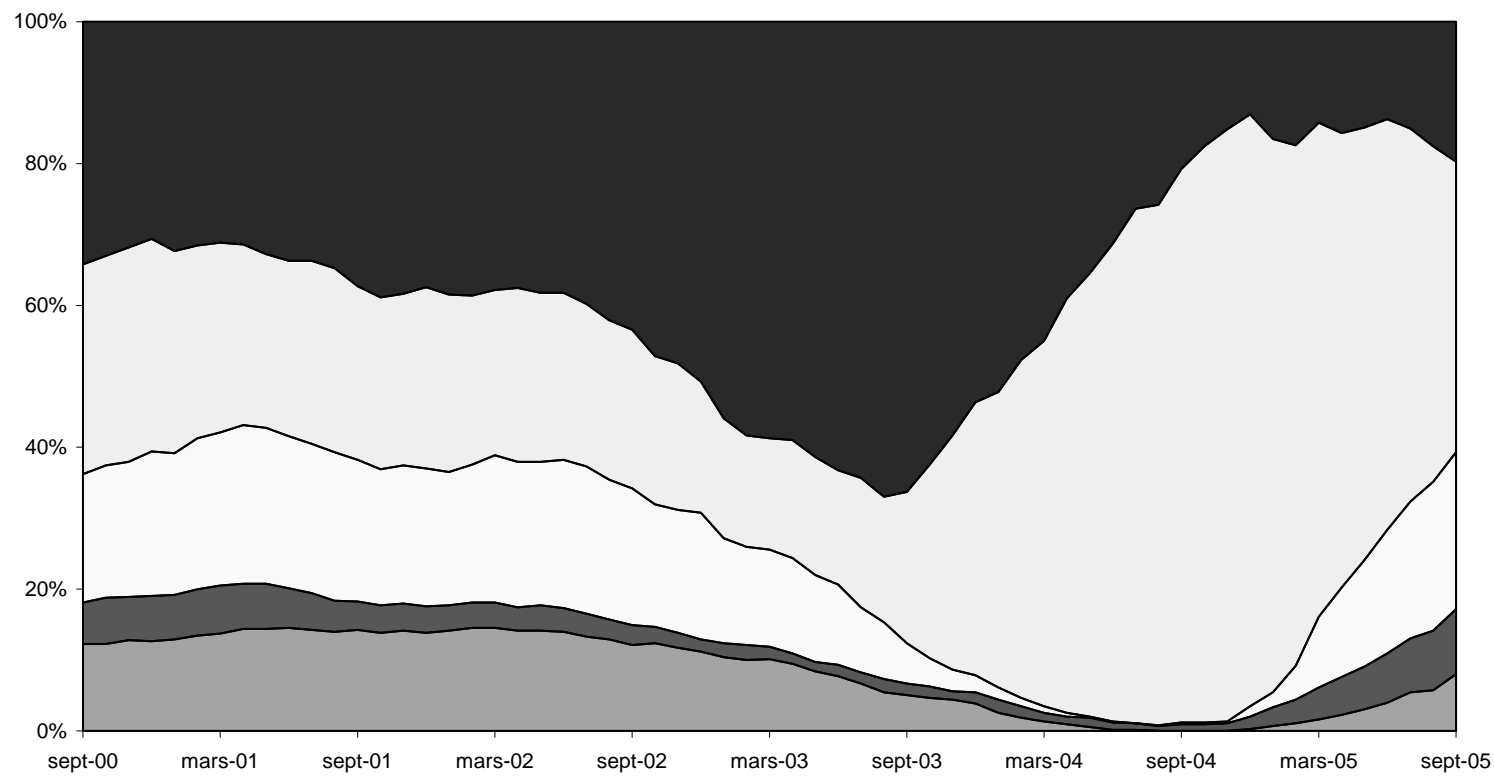

Régime 4, trajectoire 3 : $8 \%$ des parcours

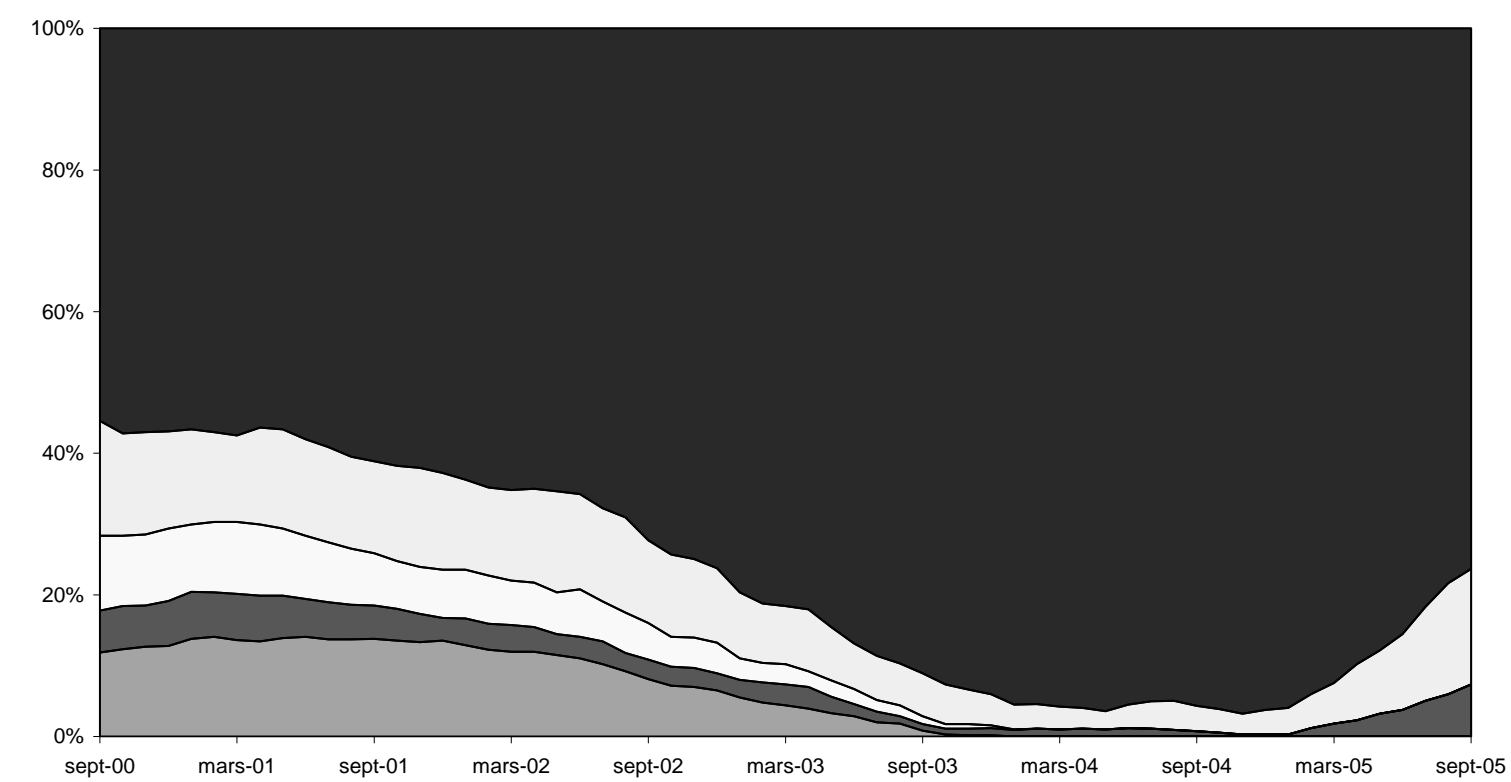

Voir légendes graphique 1.

- 40 • Travail et Emploi $n^{\circ} 113 \cdot$ Janvier-mars 2008 
comme véritable mode d'emploi et non comme simple porte d'entrée dans les entreprises, concerne une part importante de la cohorte. Alors même qu'une insuffisante flexibilité du marché du travail est souvent invoquée pour expliquer la mauvaise situation de l'emploi des jeunes en France, ces trajectoires, notamment, montrent que les modalités de recrutement alternatives au recrutement sur contrat à durée indéterminée sont largement mobilisées par les employeurs, non seulement pour les nouveaux entrants mais encore au-delà, après plusieurs années de vie active. Si deux tiers des premiers emplois des jeunes de la Génération 1998 étaient des emplois temporaires (CEREQ, 2002), le recours à ces emplois perdure par la suite puisque les premières mobilités se font encore souvent vers d'autres emplois temporaires. Le recours à ces emplois temporaires apparaît dès lors comme un outil de flexibilisation de la participation des jeunes à l'emploi.

Un second constat s'impose encore: si l'on considère que les embauches sur contrat à durée indéterminée ne signent pas toujours l'accès à un emploi durable, il apparaît que l'opposition entre continuité d'emploi et précarité ne peut se résumer à une opposition entre types juridiques d'emplois. Du point de vue ici adopté - celui des régimes temporels de la participation au marché du travail -, la prise en compte de la durabilité de la relation d'emploi permet de nuancer le propos: des individus, bien que recrutés sous contrat temporaire, parviennent à maintenir une relation continue à l'emploi pendant des années et, pour paradoxale qu'elle paraisse, cette situation suffit à infléchir un point de vue trop exclusivement centré sur la qualité du contrat de travail. Salaire et conditions de travail devraient bien sûr entrer en ligne de compte pour distinguer les emplois entre ceux qui assurent durabilité et sécurité et ceux qui maintiennent dans la précarité et l'insécurité. Mais du point de vue des temporalités, de la «galère» prévisible, vécue par de nombreux jeunes, il importe de prendre en compte d'autres facteurs susceptibles d'influer sur la durabilité de la relation d'emploi. Ainsi, certains employeurs paraissent utiliser des contrats temporaires, régulièrement renouvelés, pour assurer la permanence de service sans se lier à une main-d'œuvre pérenne. Certains jeunes n'hésitent d'ailleurs pas à tirer parti de ces circonstances. Cette situation de «précarité paradoxale» et celle, plus conforme aux représentations ordinaires, des parcours durablement discontinus nous intéresseront plus particulièrement dans la suite de cet article.

\section{Formes de l'incertitude et sécurisation des trajectoires}

Nous examinerons ici les régimes de participation au marché du travail qui témoignent soit de discontinuités manifestes de la relation d'emploi, susceptibles d'aller jusqu'au retrait plus ou moins définitif de l'emploi, soit du paradoxe d'une précarité contournée.

\section{Quand la précarité s'avère paradoxale}

Le régime 3 -celui des formes paradoxales de continuité d'emploi - présente en effet un caractère paradoxal, en des sens toutefois différents selon les deux trajectoires suivies par les individus. Dans un cas - plus de 50000 individus sont concernés ceux-ci sont, tout au long des cinq années d'observation entre 2000 et 2005 , dans cette situation surprenante qui les voit se maintenir en emploi de manière tout à fait continue bien qu'ils soient embauchés sur des contrats à durée déterminée; dans l'autre cas - plus de 60000 individus concernés - ceux-ci sont passés directement d'une situation d'emploi à durée déterminée, qui a généralement perduré plus de deux ans, à une situation d'emploi stabilisée en septembre 2005. Si cette dernière forme type de trajectoire peut s'expliquer, pour quelques individus, par des périodes de formation en alternance qui ont débouché sur une embauche durable, le paradoxe reste entier pour tous les autres et, bien entendu, dans le premier cas de figure. Tentons donc, pour commencer, de voir d'un peu plus près ce que sont ces emplois à durée déterminée longs.

\section{Paradoxes du privé, paradoxes du public}

Nous considérons ici l'emploi occupé lors du dernier mois effectué sous statut temporaire après au moins dix-huit mois passés continûment en emploi. Il ne s'agit d'emplois-jeunes que dans une minorité de cas; il s'agit le plus souvent d'emplois sous contrat temporaire ( $c f$. tableau 2), dont la durée effective excède très largement les dix-huit mois généralement autorisés par le législateur. Ces emplois sont fréquemment effectués dans le secteur public: il en va ainsi pour près des deux tiers des Edd, lorsque la trajectoire se poursuit toujours dans l'emploi temporaire en 2005 , et pour près de la moitié des Edd lorsque la trajectoire a débouché sur l'emploi pérenne. Il est vrai que le secteur public est grand consommateur d'emplois temporaires à travers le recrutement de contractuels et autres vacataires: parmi les 15-29 ans actifs dans ce secteur en 2003, $40 \%$ étaient employés sur un contrat temporaire (données tirées de l'En-

Tableau 2

Régime 3 : formes paradoxales de continuité d'emploi

\begin{tabular}{|c|c|c|c|c|}
\hline \multirow{2}{*}{$\begin{array}{l}\text { statut du } \\
\text { dernier } \\
\text { emploi } \\
\text { temporaire } \\
\text { long }\end{array}$} & \multicolumn{2}{|c|}{$\begin{array}{l}\text { trajectoires de maintien } \\
\text { dans l'emploi à durée } \\
\text { déterminée }\end{array}$} & \multicolumn{2}{|c|}{$\begin{array}{c}\text { trajectoires débouchant sur } \\
\text { un emploi pérenne }\end{array}$} \\
\hline & $\%$ & $\begin{array}{c}\text { durée } \\
\text { médiane de } \\
\text { la séquence }\end{array}$ & $\%$ & $\begin{array}{c}\text { durée } \\
\text { médiane de } \\
\text { la séquence }\end{array}$ \\
\hline Cdd & $56 \%$ & 44 mois & $52 \%$ & 29 mois \\
\hline intérim & $15 \%$ & 32 mois & $16 \%$ & 23 mois \\
\hline emploi-jeune & $18 \%$ & 58 mois & $22 \%$ & 48 mois \\
\hline autre & $11 \%$ & 33 mois & $10 \%$ & 30 mois \\
\hline
\end{tabular}


quête sur l'emploi de l'Insee, citées d'après Ire, 2005) et cette proportion n'a cessé d'augmenter depuis le début des années 1980. Si la loi n'autorise qu'un seul renouvellement d'un contrat à durée déterminée dans le secteur privé, il semble bien que les usages diffèrent sensiblement dans le secteur public: certains contractuels ou vacataires se maintiennent ainsi dans leur emploi pendant des périodes plus ou moins longues, dans l'attente d'une éventuelle titularisation. Les maîtres auxiliaires de l'Éducation nationale ou les vacataires de la recherche publique, des personnels auxiliaires des services hospitaliers ou certains employés d'administrations publiques pourraient fournir quelques-unes des figures ici repérées (10).

La continuité, au-delà de la durée légale autorisée, d'emplois sur contrat à durée déterminée dans le secteur privé surprend également. Sans doute le phénomène relève-t-il, pour une part au moins, de la diffusion de pratiques aux marges de la légalité ou, pour le moins, peu conformes au droit du travail: l'obligation de requalification des contrats à durée déterminée reconduits(11) peut être contournée, notamment, par divers artifices d'écriture. Les contrats successifs peuvent naviguer «sur le papier» entre les divers types de recours ouverts aux employeurs, qu'il s'agisse d'un changement du motif ou du service d'affectation, par exemple: ces pratiques seraient assez fréquentes (LouIT-MARTINOD, 2003). Elles peuvent, du reste, bénéficier de la relative complicité des salariés eux-mêmes qui, quitte à ne pas décrocher le contrat stable recherché, n'en obtiennent pas moins la prolongation de leur activité et la continuité de leur salaire. Par ailleurs, le délai de carence entre deux contrats à durée déterminée, variable selon la durée du contrat initial, peut aussi, lorsqu'il est de faible durée, avoir été négligé par l'individu au moment de répondre à l'enquête: celui-ci simplifie sa trajectoire professionnelle en réunissant, quand il ne les a pas oubliées, dans une seule et même séquence d'emploi des séquences trop courtes ou trop nombreuses pour être détaillées. Il en va de même lors de la déclaration des périodes d'intérim: les interruptions courtes peuvent être omises ou les missions successives, surtout chez un même employeur, réunies lors de la déclaration des périodes d'emploi. Les situations paradoxales révélées par notre analyse des trajectoires d'emploi ne sauraient toutefois être réduites à un effet de mémoires défaillantes ou de simplifications volontaires des trajectoires (12).

(10) Vacataires ou contractuels, ces agents sont généralement recrutés sur la base de contrats de droit public.

(11) Un contrat de travail à durée déterminée est susceptible d'être requalifié en contrat à durée indéterminée à la demande du salarié notamment lorsque la relation contractuelle se poursuit après l'échéance du terme.

(12) Les cas nombreux de demande de requalification du contrat de travail après plusieurs renouvellements de contrats de travail précaires, dont les Prud'hommes ont notamment à juger, témoignent à leur manière de la diffusion de situations peut-être paradoxales mais souvent réelles.

\section{Qui emprunte ce type de parcours?}

Hommes et femmes sont également présents dans ces types de trajectoires. Plus étonnant peut-être: niveau de diplôme et spécialité ne font guère de différence. Toutefois, les jeunes qui ont poursuivi des études supérieures pendant une ou deux années sans obtenir le titre visé, généralement confrontés à des difficultés d'insertion professionnelle plus grandes que les autres diplômés, sont ici quelque peu surreprésentés, en particulier parmi ceux qui restent longtemps sur un emploi temporaire avant de pouvoir stabiliser leurs conditions d'emploi. De même, les sortants des spécialités tertiaires du CAP/ BEP, souvent en difficulté sur le marché du travail pendant les premières années de leur vie active, sont un peu surreprésentés, notamment parmi ceux qui cumulent les plus longues durées d'emploi continu sur contrats à durée déterminée. Sans surprise, les individus concernés se déclarent bien plus souvent insatisfaits de leur situation professionnelle; la différence est frappante avec les individus qui ont connu l'installation dans des emplois à durée indéterminée sans anicroche notable. Ainsi, ceux qui ont connu des trajectoires paradoxales d'accès à la continuité d'emploi finissent même par apparaître deux fois plus souvent insatisfaits de leur situation en 2003 que leurs camarades ayant accédé rapidement à des Edi pérennes. Avec le temps l'incertitude sur l'avenir devient d'autant plus pesante!

En termes de salaires, les différences sont encore notables entre individus selon qu'ils ont connu une inscription rapide dans l'emploi pérenne ou une trajectoire de confinement dans l'emploi à durée déterminée. Dans ce dernier cas de figure, les salaires médians sont sensiblement inférieurs à ceux déclarés dans l'autre cas: en 2001, 940 euros contre 1220. Ces écarts se retrouvent à tous les niveaux mais ils croissent avec le niveau de diplôme: il y aurait, en quelque sorte, un coût croissant du nonaccès à l'emploi pérenne au regard du niveau de formation. En revanche, les progressions salariales des uns et des autres entre 2001 et 2005 restent relativement semblables. Entre ceux vite installés en Edi et ceux qui, après plusieurs années d'emploi(s) à durée déterminée ont finalement accédé à un emploi pérenne, la progression se fait même un peu à l'avantage des seconds, comme si un certain processus de rattrapage s'enclenchait. Ce processus apparaît gommé pour les plus diplômés, sortis de l'enseignement supérieur. Des évolutions similaires prévalent du point de vue de la catégorie socioprofessionnelle des emplois occupés.

\section{Lever l'incertitude en ajustant le contrat?}

Au-delà des écarts salariaux entre individus pourtant sortis aux mêmes niveaux de formation et des retards qu'ils induisent dans l'évolution de la carrière - ici entendue dans son sens ordinaire de progression régulière et d'amélioration constante 
de la situation professionnelle -, reste l'incertitude temporelle quant au maintien en emploi. Or la durée des périodes continues d'emploi, dans le secteur privé comme dans le secteur public, témoigne du fait que le maintien en emploi relève souvent de la reconduction immédiate ou à peine différée du contrat de travail, moins fréquemment de la signature d'un nouveau contrat pour un nouvel emploi. La levée de l'incertitude ne passerait-elle pas, dès lors, par l'ajustement du contrat de travail à la situation d'emploi effective de l'Individu? Le débat social sur l'«insécurité sociale»-selon la formule de Robert CASTEL (2003) - produite par les nouvelles situations d'emploi s'est, en effet, cristallisé autour de deux solutions types, visant pour l'une à «sécuriser le parcours professionnel» et pour l'autre à «stabiliser le contrat de travail» (LouIT-MARTINOD, 2003). Il ne s'agit pas, ici, d'abonder dans le sens de la mise en place d'un contrat de travail unique (CAhuc, Kramartz, 2004) mais de plaider pour la requalification des contrats temporaires renouvelés.

Il s'agirait en quelque sorte d'assurer l'intégration professionnelle (PAUGAM, 2000), en levant l'incertitude qui la mine, dès lors que l'activité effectuée dans le cadre d'un emploi pourvu de manière temporaire réclame, de toute évidence, d'être poursuivie, puisque le contrat en est renouvelé avec son bénéficiaire antérieur ou le poste ouvert à un nouveau recrutement. La question est ici de gestion de la main-d'œuvre: garantir la continuité d'une tâche en substituant un contrat pérenne à une succession de contrats temporaires. L'enjeu en est - en particulier lorsque les individus sont en cours d'insertion dans la vie active - de leur offrir les conditions d'une intégration professionnelle réussie plutôt que de les exposer aux risques d'une participation durablement incertaine à la vie active. L'insertion de ces individus dans les collectifs de travail en dépend (BEAUD, 1993; RAMAUX, 2006); en dépend aussi, plus largement, leur insertion sociale. $\mathrm{Si}$, dans un contexte d'accroissement des transferts familiaux entre générations, l'accès à l'autonomie résidentielle ne semble que modérément retardé par la stabilisation paradoxale sur des emplois temporaires (GoFFETTE et al., 2007), l'obtention de crédits bancaires s'en trouve souvent hypothéquée et, plus largement, la possibilité d'envisager des projets ou, plus encore, de les mettre en œuvre concrètement. C'est à des formes diverses de disqualification sociale (PAUGAM, 1991) que sont alors exposés ces individus (13).

(13) Notamment par l'impossibilité de se sentir appartenir à un groupe solidaire (PAUGAM, 1991), qu'il s'agisse du groupe des collègues de travail ou du groupe des pairs... quand ceux-ci abordent concrètement l'installation dans la vie sociale.

\section{Quand l'emploi se fait discontinu}

Les trajectoires caractéristiques du régime 4 , réunies sous le titre inscription dans l'emploi durablement instable, montrent des parcours encore moins assurés que les précédents du point de vue de l'évolution vers l'emploi pérenne. Mais la relative hétérogénéité des trajectoires au sein de ce régime nous amène à distinguer, d'une part, celles qui se déroulent loin de l'emploi, dans une sorte de repli provisoire mais prolongé, à distance de l'activité continue et, d'autre part, celles qui témoignent d'allers et venues continuels entre l'emploi et le non-emploi. Nous sommes donc conduits à explorer ici des espaces que nous qualifierons - en nous autorisant le plagiat d'une formule d'Alain SuPIOT (1999) - de «zones grises de l'insertion professionnelle des jeunes». Ces zones, marquées par des phénomènes plus ou moins rapides d'appelrejet de la main-d'œuvre sur le marché du travail (Rose, 1996), dont les jeunes font plus souvent l'expérience que leurs aînés, sont aussi caractérisées par des formes d'indétermination du comportement des jeunes eux-mêmes, coincés entre les désillusions et la nécessité de trouver malgré tout à s'employer, entre le retrait passager ou prolongé de la vie active et les efforts pour y reprendre pied (BESSES $e t$ al., 2007). Dans ces zones grises de l'insertion, le fonctionnement du marché du travail peut, en effet, produire des effets délétères.

\section{Loin de l'emploi}

Les jeunes qui suivent ces trajectoires éloignées de l'emploi - ils sont 63000 - n'ont, en moyenne, passé que vingt-trois mois en emploi, contre trentesept au chômage, dix-neuf en inactivité et quatre en formation au cours des sept années écoulées entre 1998 et $2005 .$. Soit à peine un peu plus du quart de leur temps en activité salariée! Et cette proportion baisse encore si l'on ne prend en compte que la période 2000-2005. Ces constats peuvent induire l'idée selon laquelle une partie de ces situations pourrait être imputée à des jeunes femmes qui se retirent de l'activité pour élever leurs enfants. Or, si les jeunes femmes sont bel et bien surreprésentées dans ce groupe puisqu'elles en constituent les deux tiers, fréquemment peu ou pas diplômées, leur retrait relatif de l'emploi ne semble pas pouvoir être réduit au souci d'élever les enfants... à moins de considérer que les femmes peu formées se comportent «naturellement» d'une manière différente de celles qui sont entrées sur le marché du travail avec des titres scolaires plus élevés! Leur retrait doit certainement être relié à la faiblesse de leur capital scolaire, qui les conduit à s'adapter différemment à la situation sur le marché du travail, c'est-à-dire à arbitrer entre 
une carrière professionnelle aléatoire et le soin des enfants au profit de cette seconde option(14).

Ainsi, le bas niveau de formation des individus de Génération 1998 qui suivent ce type de trajectoires paraît bien désigner la raison principale de leur situation, sinon de leurs difficultés, sur le marché du travail. Outre la faiblesse de ce niveau de formation, comparé avec celui de leurs camarades, environ quatre individus sur dix n'ont absolument aucun diplôme à faire valoir - soit parce qu'ils sont sortis de l'enseignement général sans titre, soit parce qu'ils n'ont pas mené à son terme la préparation d'un CAP/BEP - et qu'ils ont bien souvent suivi une formation de type tertiaire, moins efficace dans l'accès à l'emploi, même pour ceux qui ont obtenu leur diplôme. Insuffisamment formés, ces individus tirent aussi un faible parti des occasions de formation postscolaire : seulement un quart d'entre eux ont suivi une formation durant les sept années écoulées depuis la fin de leur scolarité initiale, le plus souvent de courte durée. L'accès aux formations ouvertes à ces jeunes en déshérence semble d'ailleurs réclamer la mise en œuvre de certaines compétences qui leur font défaut, bien qu'elles apparaissent indispensables pour affronter les sélections à l'entrée des formations qualifiantes; ceux qui peinent à formuler un projet, par exemple, ou, plus banalement, ceux que les distances géographiques effraient et tiennent loin des formations proposées (SAUVAGEOT et al., 2005) risquent ainsi de rester éloignés de l'emploi comme des dispositifs d'accompagnement.

\section{Aller et venir entre emploi et non-emploi}

Environ 75000 jeunes de la génération observée - soit $10 \%$ de son effectif total - ont suivi des trajectoires d'emploi très discontinues, c'est-àdire marquées par des interruptions plus ou moins longues de l'activité entre deux emplois successifs. S'ils se tiennent, globalement, moins loin de l'emploi que ceux ou celles qui ont suivi les trajectoires précédentes, ils n'en doivent pas moins faire assaut de volonté pour, bon an mal an, se maintenir en activité. Leurs parcours se caractérisent en effet par un régime de participation au marché du travail sous le sceau d'une alternance de périodes d'emploi et de non-emploi, d'autant plus rapide que les emplois sont plus courts et les périodes de non-emploi plus brèves. Ainsi, le régime de participation à l'emploi prend ici en compte non seulement les durées des emplois successifs ou leur durée cumulée mais aussi la rapidité avec laquelle les individus alternent emploi et non-emploi. En l'occurrence, les deux tiers environ des individus impliqués ont connu au moins quatre séquences d'emploi successives entre 1998 et 2005 , chacune d'une durée médiane inférieure à une année. Seulement un quart d'entre eux a cumulé

(14) Certaines aides, telles l'allocation parentale d'éducation, peuvent aussi jouer un rôle incitatif dans cet arbitrage. moins de deux années d'activité salariée au cours de la période et, s'ils ont, en moyenne, passé plus de cinquante mois en emploi, les épisodes d'emploi successifs n'en restent pas moins brefs.

Les jeunes femmes ne sont que légèrement surreprésentées parmi les individus qui suivent ces trajectoires et c'est plutôt le faible niveau de formation qui, ici encore, rassemble les unes et les autres. Les jeunes démunis de tout diplôme sont, en effet, deux fois plus nombreux que dans la génération entière: $30 \%$ d'entre eux ne disposent d'aucun titre scolaire. Les non-diplômés sont donc un peu moins nombreux que dans la population précédente, celle qui s'engage dans les trajectoires les plus éloignées de l'emploi. Mais il y a plus étonnant: environ 20\% d'entre eux détiennent un diplôme de l'enseignement supérieur. Ces parcours heurtés ne tiennent donc pas exclusivement à l'absence de titre scolaire: l'inadaptation de la formation suivie aux exigences du marché du travail, notamment, joue un rôle et, sans doute, certains jeunes diplômés suivent-ils des trajectoires qui les amènent à explorer diverses situations d'emploi avant de se déterminer de manière plus définitive. Quoi qu'il en soit, ces trajectoires exigent des efforts pour retourner vers l'emploi après en avoir été écarté pendant un temps. Dans certains cas d'ailleurs, ces efforts débouchent sur l'emploi plus durable, soit de manière paradoxale ( $c f$. ci-dessus), par l'accès à des emplois temporaires longs, ou plus traditionnellement par l'accès à des emplois sous contrat à durée indéterminée.

\section{Sécuriser les parcours d'insertion?}

Ces deux types de trajectoires d'emploi hésitantes illustrent crûment les «zones grises» de l'insertion professionnelle: aux périodes d'emploi plus ou moins fugaces succèdent des périodes de recherche d'emploi plus ou moins laborieuses et, lorsqu'il faut bien "faire avec» la situation sur le marché du travail, les retraits de l'activité salariée peuvent relever autant du découragement ou de l'épuisement que d'arbitrages sous contrainte. Les retours en activité résultent, quant à eux, d'une constance à chercher une place satisfaisante dans la vie active sinon de la dure nécessité de gagner sa vie. Si la variabilité des solutions trouvées par les unes ou les autres tient ainsi à la diversité des modalités individuelles d'adaptation aux «turbulences d'emploi» (Nicole-DrancourT, 1992) - dont l'arbitrage entre engagement dans l'activité et retrait pour élever les enfants ne fournit qu'un exemple - les allers et retours entre l'emploi et le non-emploi n'en démontrent pas moins les difficultés pour atteindre une insertion professionnelle durable, sur laquelle s'appuyer pour s'engager dans une vie adulte ( $c f$. cas des jeunes ouvriers, ECKERT, 2006). Comment lever, alors, les risques qui pèsent sur ces trajectoires et éviter des formes d'éloignement du marché du travail qui résultent finalement moins d'une éventuelle volonté de retrait que de la «vulnérabilité» 
(CASTEL, 1995) des individus dans une situation d'emploi défavorable?

Si la flexicurité vise l'assouplissement des règles de conclusion ou de rupture du contrat de travail tout en garantissant le salarié contre la perte de son salaire et de ses avantages entre deux périodes d'emploi (Louit-MARTINOD, 2003), l'enjeu paraît ici davantage de parer aux risques de décrochage de l'emploi encourus par ces jeunes adultes qui peinent à retrouver une activité salariée, c'est-à-dire aux risques de marginalisation, voire d'exclusion de la vie sociale courante. Il ne s'agit plus alors d'assurer seulement les périodes de non-emploi entre deux séquences d'emploi effectif mais de favoriser une continuité susceptible de garantir contre les risques potentiels de dérive. En ce sens, il s'agirait plutôt de favoriser une solution inspirée de la proposition, avancée pour la première fois dans le «rapport BoIsSONNAT» (1995), de «contrat d'activité» : «élargir le cadre d'organisation de la relation d'emploi» de manière à garantir sa durabilité dans un système d'obligations réciproques entre les jeunes en recherche d'une stabilisation professionnelle et les entreprises susceptibles de leur fournir une plus grande continuité d'emploi. Les périodes éloignées de l'emploi pourraient alors permettre aux jeunes de compléter leur formation professionnelle insuffisante ou inadaptée (15). Dans un tel contexte, l'adhésion des jeunes à l'effort de formation se trouverait sans doute facilitée.

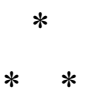

Sans doute la remise en cause du compromis salarial hérité des Trente glorieuses, a-t-elle suscité des contextes d'emploi dans lesquels «la probabilité d'être contraint de changer de situation est forte.» (FouRCADE, 1992) Si cette définition désigne précisément la "précarité» à laquelle sont, de plus en plus souvent, confrontés des catégories de plus en plus larges de salariés jusque-là "intégrés» (Paugam, 2000), elle paraît moins satisfaisante dès lors qu'il s'agit d'insertion professionnelle et sociale des jeunes: l'incertitude ne résulte pas, en l'occurrence, d'un aléa. Elle est donnée d'emblée comme une épreuve à surmonter pour ceux qui ne savent que trop bien qu'ils vont devoir, à l'instar de leurs aînés, "galérer» pour accéder à une situation pérenne sur le marché du travail et obtenir la reconnaissance sociale qui l'accompagne. Suffit-il alors de considérer la «précarité» comme la caractéristique d'une situation? Ne faut-il pas, au contraire, la

(15) Pour une évaluation de ces solutions, voir ZimmermanN, 2006. poser comme l'effet d'un rapport social, qui lie les jeunes concernés avec les générations antérieures?

L'analyse des régimes de participation des jeunes au marché du travail nous permet, nous semble-t-il, d'avancer dans cette direction: le statut d'emploi n'est pas ici seul en jeu puisque, paradoxalement, certains individus réussissent à se maintenir continûment en emploi bien qu'ils ne bénéficient que de contrats temporaires. Reste, toutefois, que les contrats temporaires en maintiennent d'autres éloignés de l'emploi continu malgré leur apparente détermination à y accéder. Ainsi, tout se passe comme si la multiplication des formes d'emploi dérogatoires à l'emploi sur contrat à durée indéterminée, visait à créer cette "zone grise» à la périphérie de l'emploi continu, la discontinuité de la relation d'emploi permettant l'ajustement des volumes d'emploi offerts et des demandes d'emploi sur le marché du travail, au détriment d'une part variable de ceux qui se voient proposer des contrats temporaires (16). Ce mode d'ajustement - en tant qu'il dépend de la diversification des types de contrat de travail proposés, bien que cette diversification soit généralement présentée comme une réponse aux difficultés d'accès à l'emploi de certaines catégories sociales - renvoie in fine à un rapport intergénérationnel: l'ajustement de l'offre et de la demande d'emploi se fait au détriment des nouveaux venus.

Si le rapport générationnel tend à s'inverser pour les travailleurs plus âgés (17), au bénéfice cette fois des générations qui leur sont postérieures, la précarité vise aussi à induire l'acceptation d'une situation salariale que l'individu n'aurait pas souhaitée d'emblée. C'est depuis ce point de vue que Michel Pialoux (1979) analysait le rapport des jeunes de banlieue au travail intérimaire dans les années soixante-dix. Tout juste faudrait-il ajouter que la panoplie des formes d'emploi s'est amplifiée alors que les opportunités d'emploi se sont encore réduites, du moins en termes relatifs. Car la précarité a effectivement partie liée avec la pauvreté. Dans une étude sur le chômage restée célèbre, Raymond LEDRUT (1966) notait déjà que, «le vrai chômeur, qui reste longtemps au chômage ou qui y retombe

(16) Examinons un cas de figure fictif! Si des emplois à durée déterminée étaient proposés en quantité telle que tout individu puisse aller de l'un de ces emplois vers un autre sans risquer le chômage, l'aléa disparaîtrait de fait: excepté quelques difficultés d'ajustement circonstancielles, les individus écartés de l'emploi sur contrat à durée indéterminée parviendraient, dans une telle situation, à se maintenir continûment en emploi. Sans vouloir demander à la comptabilité de l'emploi plus qu'elle ne peut donner, il n'en apparaît pas moins que la rareté globale de l'emploi constitue l'autre condition de l'aléa qui fonde la précarité de certains individus dans les sociétés salariales et conduit à la nécessité de trouver certains ajustements, au détriment des plus jeunes notamment.

(17) À l'autre bout du cycle de vie, en effet, certains travailleurs âgés se retrouvent confrontés à la précarité, dès lors qu'ils doivent rechercher un emploi pour sortir du chômage. 
souvent, constitue un groupement particulièrement paupérisé.» Nous relèverons surtout, dans cette citation, le fait que l'auteur évoque, outre le chômeur de longue durée, ces individus qui retombent souvent au chômage: or c'est bien le cas de ces jeunes qui sont soumis aux mécanismes d'appelrejet sur le marché du travail et qui oscillent entre participation active à l'emploi, chômage récurrent, voire retrait plus ou moins durable de l'emploi.

Une analyse de la relation salariale dans laquelle entrent les nouveaux venus sur le marché, en particulier ceux qui restent à la périphérie de l'emploi continu, pourrait ainsi former l'autre versant de l'analyse de la précarité chez les jeunes que nous venons de proposer. Nous avons délibérément choisi de nous en tenir ici aux aspects temporels de la relation d'emploi : la description des régimes de participation au marché du travail de ces jeunes nous a permis de montrer comment une dynamique individuelle est connectée avec une dynamique sociale et en quoi la dimension intergénérationnelle est nécessaire pour comprendre les formes d'exclusion relative de certains jeunes de l'emploi durable. Sans doute certains se trouvent-ils aussi exclus d'une continuité de revenu et réduits à la position d'outsiders dans une société salariale qui se lézarde. L'observation des régimes de revenu constitue ainsi l'autre face de l'analyse des régimes de participation temporelle au marché du travail.

\section{Bibliographie}

Beaud S., (1993), «Le rêve de l'intérimaire», in P. Bourdieu (dir.), La misère du monde, Seuil, Paris.

BESSES M. DE et al., (2007), «Une formation qualifiante différée pour les jeunes non diplômés? Un éclairage à partir du cheminement professionnel de jeunes sortis du système éducatif en 1998 », Cereq, Relief, $\mathrm{n}^{\circ} 23$, Marseille.

Boissonnat J., (1995), Le travail dans vingt ans, Éditons Odile Jacob / La Documentation française, Paris.

Cahuc P., Kramartz F., (2004), De la précarité à la mobilité: vers une sécurité sociale professionnelle, La Documentation française, Paris.

CAStel R., (1995), Métamorphoses de la question sociale, une chronique du salariat, Fayard, Paris.

Castel R., (2003), L'insécurité sociale, Seuil, Paris.

Cereq, (2002), Quandl'école est finie... Premiers pas dans la vie active de la Génération 1998, Cereq, Marseille.

Cereq, (2005), Quandl'école est finie... Premiers pas dans la vie active de la Génération 2001, Cereq, Marseille.

CEREQ, (2007), Quand la carrière commence, les sept premières années de la vie active de la Génération 1998, Cereq, Marseille.

DuBEt F., (1987), La galère: jeunes en survie, Fayard (et Points Seuil), Paris.

ECKert H., (2006), Avoir vingt ans à l'usine, La Dispute, Paris.

FourCADE B., (1992), «L'évolution des situations d'emploi particulières de 1945 à $1990 »$, Travail et Emploi, $\mathrm{n}^{\mathrm{O}} 52$.

Goffette J., Mora V., Sulzer E., (2008), Insertion professionnelle et autonomie résidentielle des jeunes, rapport de l'ONPES, à paraître.

Ires, (2005), Les mutations de l'emploi en France, La Découvertes, coll. «Repères », Paris.

Leclerc-Olive M., Engrand S., (2000), «Sortir de la précarité de l'emploi: entre routine et projet», in
Billiard I., Debordeaux D. et Lurol M., Vivre la précarité, trajectoires et projets de vie, L'Aube, La Tour d'Aigues.

Ledrut R., (1966), Sociologie du chômage, PUF, Paris.

Lochet J.-F., (1994), Logiques d'usage des statuts d'embauche des jeunes en insertion (le cas des jeunes de niveau VI et V de formation), broché, Cereq, Marseille.

Louit-MARTINOD N., (2003), L'évolution contemporaine $d u$ droit des contrats de travail à durée limitée, Presses universitaires d'Aix-Marseille.

Maruani M., Reynaud E., (2004 pour la dernière éd.), Sociologie de l'emploi, La Découverte, coll. «Repères », Paris.

Nicole-Drancourt C., (1992), «L'idée de précarité revisitée», Travail et Emploi, $\mathrm{n}^{\circ} 52$.

Paugam S., (1991), La disqualification sociale, essai sur la nouvelle pauvreté, PUF, Paris.

Paugam S., (2000), Le salarié de la précarité, les nouvelles formes de l'intégration professionnelle, PUF, Paris.

PIAloux M., (1979), «Jeunesse sans avenir et travail intérimaire», Actes de la recherche en sciences sociales, $\mathrm{n}^{\circ}$ 26-27, mars-avril.

Ramaux C., (2006), Emploi : éloge de la stabilité - L'État social contre la flexicurité, Éditions Mille et une nuits, Paris.

Rose J., (1996), «L'organisation des transitions professionnelles: entre socialisation, mobilisation et recomposition des rapports de travail et d'emploi », Sociologie $d u$ travail, $\mathrm{n}^{\mathrm{o}} 1$.

SAuvageot et al., (2005), "Quelle formation qualifiante différée pour les jeunes non diplômés?», Note d'évaluation de la DEP, 05.01, mars.

Supiot A., (1999), Au-delà de l'emploi, Flammarion, Paris.

ZimmermannB.,(2006), «Les groupementsd'employeurs : la sécurité dans la flexibilité?», Sociologie du travail, 48 (1). 